\title{
HIGH-RESOLUTION MASS MODELS OF DWARF GALAXIES FROM LITTLE THINGS
}

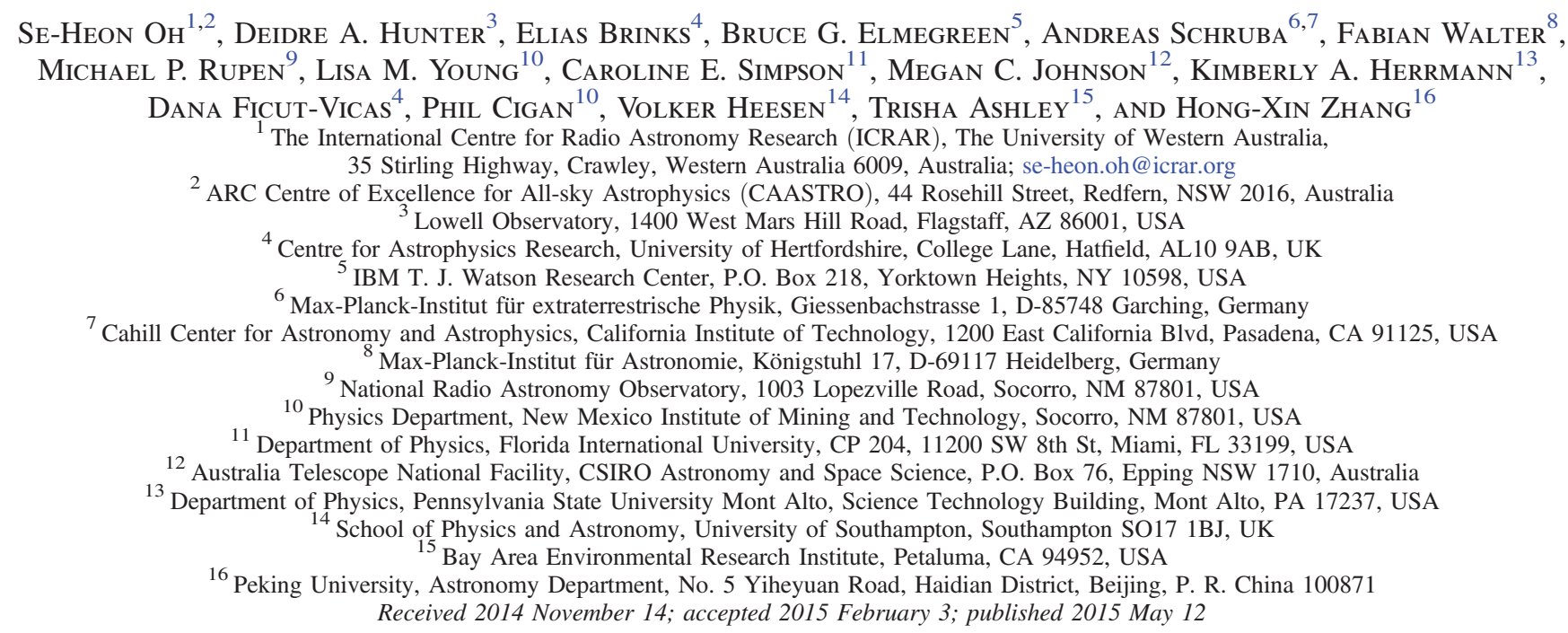

\begin{abstract}
We present high-resolution rotation curves and mass models of 26 dwarf galaxies from "Local Irregulars That Trace Luminosity Extremes, The H I Nearby Galaxy Survey" (LITTLE THINGS). LITTLE THINGS is a high-resolution $\left(\sim 6^{\prime \prime}\right.$ angular; $<2.6 \mathrm{~km} \mathrm{~s}^{-1}$ velocity resolution) Very Large Array $\mathrm{H}$ i survey for nearby dwarf galaxies in the local volume within $11 \mathrm{Mpc}$. The high-resolution $\mathrm{H}_{\text {I }}$ observations enable us to derive reliable rotation curves of the sample galaxies in a homogeneous and consistent manner. The rotation curves are then combined with Spitzer archival $3.6 \mu \mathrm{m}$ and ancillary optical $U, B$, and $V$ images to construct mass models of the galaxies. This high quality multi-wavelength data set significantly reduces observational uncertainties and thus allows us to examine the mass distribution in the galaxies in detail. We decompose the rotation curves in terms of the dynamical contributions by baryons and dark matter (DM) halos, and compare the latter with those of dwarf galaxies from THINGS as well as $\Lambda$ CDM Smoothed Particle Hydrodynamic (SPH) simulations in which the effect of baryonic feedback processes is included. Being generally consistent with THINGS and simulated dwarf galaxies, most of the LITTLE THINGS sample galaxies show a linear increase of the rotation curve in their inner regions, which gives shallower logarithmic inner slopes $\alpha$ of their DM density profiles. The mean value of the slopes of the 26 LITTLE THINGS dwarf galaxies is $\alpha=-0.32 \pm 0.24$ which is in accordance with the previous results found for low surface brightness galaxies $(\alpha=-0.2 \pm 0.2)$ as well as the seven THINGS dwarf galaxies $(\alpha=-0.29 \pm 0.07)$. However, this significantly deviates from the cusp-like DM distribution predicted by DM-only $\Lambda$ CDM simulations. Instead our results are more in line with the shallower slopes found in the $\Lambda$ CDMSPH simulations of dwarf galaxies in which the effect of baryonic feedback processes is included. In addition, we discuss the central DM distribution of DDO 210 whose stellar mass is relatively low in our sample to examine the scenario of inefficient supernova feedback in low mass dwarf galaxies predicted from recent $\Lambda$ CDM SPH simulations of dwarf galaxies where central cusps still remain.
\end{abstract}

Key words: dark matter - galaxies: dwarf - galaxies: halos - galaxies: irregular - galaxies: kinematics and dynamics

Supporting material: data behind figures

\section{INTRODUCTION}

Dark matter (DM), the existence of which is indirectly invoked by its gravitational effect in individual galaxies as well as in galaxy clusters, dominates, together with dark energy, the energy budget in the universe (Zwicky 1937; van den Bergh 1961; Rubin \& Ford 1970; Bosma 1978; Peebles 1982; Riess et al. 1998; Perlmutter et al. 1999; Spergel et al. 2003). In particular, the role of DM is critical not only in forming and evolving galaxies in the early universe but also in shaping the large-scale structure in the universe through cosmic time (Blumenthal et al. 1984; Colless et al. 2001a; Padmanabhan et al. 2007; Jones et al. 2009; Komatsu et al. 2011, etc.). The cosmological importance of DM has driven efforts to explore the physical nature of DM particles and to attempt their direct detection (Moore et al. 2001; Gaitskell 2004; Angloher et al. 2012; Akerib et al. 2014, and references therein). Of many candidates for DM particles, Cold Dark Matter (CDM) has been envisaged as one of the most successful models in that numerical simulations based on a paradigm combining CDM with the cosmological constant, $\Lambda$ (so-called $\Lambda \mathrm{CDM}$ ). This describes well the large-scale structure in the universe traced by surveys such as the $\operatorname{SDSS}^{17}$ (York et al. 2000; Doroshkevich

\footnotetext{
17 The Sloan Digital Sky Survey (http://www.sdss.org/).
} 
et al. 2004), $2 \mathrm{dFGRS}^{18}$ (Colless et al. 2001b), 6dFGS ${ }^{19}$ (Jones et al. 2004), and CMB power spectrum observations (Primack 2003; Spergel et al. 2003, 2007; Planck Collaboration et al. 2014).

However, despite the success of cosmological $\Lambda \mathrm{CDM}$ simulations in producing the large-scale structure of the universe, distinct differences between the simulations and observations have been found in the DM distribution near the center of individual galaxies. The simulations have consistently predicted cusp-like DM distributions toward the centers of galaxies, being described by a power law, $\rho \sim R^{\alpha}$ where $R$ is the galaxy radius and $\alpha \sim-1.0$ (Moore 1994; Navarro et al. 1996b, 1997, 2004a, 2010; Moore et al. 1999; Ghigna et al. 2000; Klypin et al. 2001; Power et al. 2003; Stoehr et al. 2003; Diemand et al. 2008; Stadel et al. 2009; Ishiyama et al. 2013, etc.). In contrast, inferred DM distributions in nearby dwarf galaxies in the local universe have shown a linear velocity increase toward their centers, giving rise to a sizable density-core $\left(\rho \sim R^{\alpha}\right.$ where $\left.\alpha \sim 0.0\right)$ (Moore 1994; de Blok et al. 1996, 2001; de Blok \& McGaugh 1997; de Blok \& Bosma 2002; Weldrake et al. 2003; Spekkens et al. 2005; Kuzio de Naray et al. 2006, 2008; Oh et al. 2008, 2011b, 2011a; see, however, Adams et al. 2014 for a discussion on gas kinematics which produces shallower density profiles than those from stellar kinematics). This clear discrepancy of the central DM distribution in galaxies between $\Lambda$ CDM simulations and observations, the so-called "cusp/core" problem, has been one aspect of the small-scale crisis in $\Lambda$ CDM cosmology which is likely connected to the "missing satellites" problem (Brooks et al. 2013) and "too-big-to-fail" problem (dense satellites; see Boylan-Kolchin et al. 2011, 2012). Given that DM combined with the $\Lambda$ CDM paradigm is successful in explaining both the large-scale structure in the universe as well as galaxy formation and evolution, there are good reasons to explore ways to resolve the "cusp/core" problem. We refer to de Blok (2010) and Pontzen \& Governato (2014) for the latest review of the "cusp/core" problem.

Late-type dwarf galaxies in the local universe, with a simple dynamical structure (no bulge and spiral components) have been used for addressing the central DM distribution in galaxies since their dynamics are usually dominated by DM, enabling us to derive more accurately the DM distribution near their centers. These diffuse dwarf galaxies have provided significant observational constraints on the central DM distribution in galaxies. Over the past decade, several highresolution neutral hydrogen $\left(\mathrm{HI}_{\mathrm{I}}\right)$ surveys of galaxies in the local universe $(<11 \mathrm{Mpc})$ using radio interferometers, among others The H i Nearby Galaxy Survey (THINGS) ${ }^{20}$ (Walter et al. 2008), Very Large Array (VLA)-ANGST ${ }^{21}$ (Ott et al. 2012), Local Irregulars That Trace Luminosity Extremes, THINGS (LITTLE THINGS) ${ }^{22}$ (Hunter et al. 2012), FIGGS $^{23}$ (Begum et al. 2008), SHIELD $^{24}$ (Cannon et al.

\footnotetext{
18 The 2dF Galaxy Redshift Survey (http://www2.aao.gov.au/2dfgrs/).

19 The 6dF Galaxy Survey (http://oldweb.aao.gov.au/local/www/6df/).

20 The H I Nearby Galaxy Survey (http://www.mpia-hd.mpg.de/THINGS/ Overview.html).

21 VLA-ACS Nearby Galaxy Survey Treasury (https://science.nrao.edu/ science/surveys/vla-angst).

22 Local Irregulars That Trace Luminosity Extremes, The H I Nearby Galaxy Survey (https://science.nrao.edu/science/surveys/littlethings).

23 Faint Irregular Galaxies GMRT Survey.

24 The Survey of HI in Extremely Low-mass Dwarfs (http://www.macalester. edu/ jcannon/shield.html).
}

2011), LVHIS ${ }^{25}$ (Koribalski 2010), have allowed us to derive more reliable $\mathrm{H}$ I rotation curves of galaxies and examine their central mass distributions within $1 \mathrm{kpc}$ where the predictions of $\Lambda \mathrm{CDM}$ simulations are most distinctive. For example, highresolution $(0.1-0.2 \mathrm{kpc})$ DM density profiles of seven dwarf galaxies from THINGS, complemented with the "Spitzer Infrared Nearby Galaxies Survey" (SINGS; Kennicutt et al. 2003), were derived by Oh et al. (2008, 2011b). From this, they found that the mean value of the inner density slopes, $\alpha$, of the seven dwarf galaxies is $-0.29 \pm 0.07$ which is in good agreement with the value of $-0.2 \pm 0.2$ derived earlier from a larger number of Low Surface Brightness (LSB) galaxies (de Blok \& Bosma 2002). In the past, it has been argued that observational systematic effects such as beam smearing, center offsets, and non-circular motions could have affected the derived central DM distributions of galaxies as these observational biases tend to flatten the derived inner DM density profiles, hiding the central cusps. However, because of the high resolution and quality of the above mentioned observations, these potential biases were significantly reduced. As a result, observational evidence for the corelike distribution of DM near the centers of dIrr galaxies is particularly strong.

In order to explain this behavior, baryonic feedback processes have been proposed as a means for removing the central cusps expected from $\Lambda$ CDMDM-only simulations. More specifically, it is expected that DM and baryons in galaxies can be substantially redistributed by frequent explosions of supernovae (SNe) (Larson 1974; Navarro et al. 1996a; Dekel et al. 2003; Mo \& Mao 2004; Mashchenko et al. 2008; de Souza et al. 2011; Brook et al. 2011; Pontzen \& Governato 2012; di Cintio et al. 2014; Ogiya \& Mori 2014, etc.). However, due to numerical difficulties in simulating multi-phase gas physics as well as the lack of understanding of the detailed baryonic physics in galaxies, taking the baryonic feedback into account in hydrodynamical simulations of dwarf galaxies was considered difficult and limited to simulations of high-redshift galaxies (e.g., Mashchenko et al. 2006).

Governato et al. (2010) were the first to perform highresolution cosmological $\mathrm{N}$-body+Smoothed Particle Hydrodynamic (SPH) simulations of dwarf galaxies which include the effect of detailed baryonic feedback processes, in particular physically motivated gas outflows driven by SN explosions. From this, they found that the photometric and kinematic properties of the simulated dwarf galaxies are in close, qualitative agreement with those of observed nearby dwarf galaxies. More quantitatively, Oh et al. (2011a) performed an analysis of the baryonic and DM mass distributions of the simulated dwarf galaxies, in exactly the same way as the THINGS dwarf galaxies were analyzed, and showed that their derived rotation curves and the corresponding DM density profiles are consistent with those of the THINGS dwarf galaxies and show a linear increase of velocity in the inner region inherent of shallow DM density profiles. This suggests that repeated gas outflows driven by $\mathrm{SN}$ explosions even without a burst of star formation are able to play a fundamental role in removing the central cusps and inducing flatter DM density slopes near the centers of dwarf galaxies. This is in contrast to clusters of galaxies where galaxy interactions are more likely to be the dominant mechanisms for the removal of

25 The Local Volume HI Survey (http://www.atnf.csiro.au/research/LVHIS/). 
central cusps rather than star formation activities (Richtler et al. 2011). See Governato et al. (2010) and Pontzen \& Governato $(2012,2014)$ for detailed discussions regarding the effect of SN explosions on the central cusps.

THINGS was only able to probe a small number (i.e., seven) of dwarf galaxies. It is therefore essential to extend the investigation to a larger number of dwarf galaxies in order to obtain a statistically robust observational sample to which simulations can be compared. Data on a larger sample of dIrr galaxies have now been provided by the latest $\mathrm{H}_{\mathrm{I}}$ survey of nearby galaxies, LITTLE THINGS (Hunter et al. 2012). LITTLE THINGS is a high-resolution ( $\sim 6^{\prime \prime}$ angular; $\leqslant 2.6$ $\mathrm{km} \mathrm{s}^{-1}$ velocity resolution) $\mathrm{H}_{\mathrm{I}}$ survey for 41 nearby $(<11 \mathrm{Mpc})$ gas-rich dwarf galaxies undertaken with the NRAO $^{26}$ VLA in the northern sky. The $\mathrm{H}_{\mathrm{I}}$ observations are complemented with other wavelength data, such as $\mathrm{H} \alpha$, optical $U, B, V$, and near-infrared (Hunter \& Elmegreen 2006), archival Spitzer infrared, and GALEX ultraviolet images as well as follow-up observations with ALMA and Herschel. These high-quality multi-wavelength data sets significantly reduce the observational uncertainties inherent in low resolution data which may result in hiding the central cusps. Of the 41 galaxies, we select a sample of 26 dwarf galaxies (three of them are also in THINGS) which show a regular rotation pattern in their velocity fields. In this paper, we extract (1) bulk and non-circular motions of the sample galaxies, (2) derive rotation curves, (3) decompose the derived rotation curves in terms of the contributions by baryons and DM halos, and (4) address the central DM distribution by making a direct comparison between the derived DM distributions of the galaxies to those of $\mathrm{SPH}+N$-body simulations of dwarf galaxies.

The structure of this paper is as follows. The data used for deriving the mass models of our sample galaxies are described in Section 2. In Section 3, we present the rotation curves, the mass models of baryons, and the DM mass modeling of the galaxies. In Section 4, we discuss the central DM distributions of the sample galaxies by comparing them with those of dwarf galaxies from both THINGS and simulations. We then discuss the effect of SN feedback on the central cusp in Section 5, followed by the discussion of the effect of beam smearing on the central DM distribution of galaxies in Section 6. Lastly, we summarize the main results of this paper and conclusions in Section 7.

\section{THE DATA}

We use high-resolution $\mathrm{H}_{\mathrm{I}}$ data of 26 nearby $(<11 \mathrm{Mpc})$ dwarf galaxies from LITTLE THINGS to address the central DM distribution of the galaxies. The sample galaxies show a regular rotation pattern in their two-dimensional (2D) $\mathrm{HI}$ velocity fields (see appendix), allowing us to derive reliable rotation curves which include the contributions to their kinematics of both their DM halo, i.e., non-baryonic and baryonic matter. Considering the distances of the sample galaxies, the linear resolutions of the LITTLE THINGS H I data

\footnotetext{
${ }^{26}$ NRAO is a facility of the National Science Foundation operated under cooperative agreement by Associated Universities, Inc. These data were taken during the upgrade of the VLA to the Expanded VLA or EVLA. In this paper we refer to the instrument as the VLA, the retrofitted antennas as EVLA antennas, and non-retrofitted antennas as VLA antennas. This emphasizes the hybrid nature of the instrument and distinguishes it from the far more powerful Jansky VLA or JVLA it has become since 2012.
}

$\left(\sim 6^{\prime \prime}\right)$ range from $\sim 26$ to $200 \mathrm{pc}$ with an average of $100 \mathrm{pc}$ which is sufficient to resolve the inner $1 \mathrm{kpc}$ region of the galaxies in so far unmatched detail. This enables us to examine the central DM distributions of the galaxies in detail. In addition, observational systematic effects inherent in lowresolution data (e.g., beam smearing, kinematic center offset and non-circular motions) are significantly reduced in the highresolution $\mathrm{H}_{\mathrm{I}}$ data, which allows us to derive more accurate underlying kinematics of the sample galaxies.

Although the total kinematics of late-type dwarf galaxies is dominated by DM (Prada \& Burkert 2002), it is nonetheless important to separate the contribution by baryons from the total rotation curve. This is achieved by using Spitzer archival IRAC $3.6 \mu \mathrm{m}$ and ancillary optical color information (Hunter $\&$ Elmegreen 2006). Spitzer IRAC $3.6 \mu \mathrm{m}$ images are much less affected by dust than maps at shorter wavelengths and trace the old stellar populations that occupy the dominant fraction of the stellar mass in galaxies (Walter et al. 2007). Although there is some contamination by polycyclic aromatic hydrocarbon emission extending into the $3.6 \mu \mathrm{m}$ band (Meidt et al. 2014) this is reduced in dIrr galaxies which, by virtue of having low heavy element abundances, have a correspondingly lower dust content.

According to the simulations by Governato et al. (2012), the degree of baryonic feedback is largely dependent on the amount of stars, so past star formation activity in the sense that for the same total mass budget (baryons + DM), those galaxies with a higher past star formation activity will have had more significant outflows. Therefore a reliable measurement of the stellar mass in a galaxy is essential to investigating the effect of baryonic feedback on the central cusp. The basic observational properties of the sample galaxies are listed in Table 1. We refer to Hunter et al. (2012) for a complete description of the $\mathrm{H}_{\mathrm{I}}$ observations and data reduction.

\section{MASS MODELS}

In this section, we perform mass modeling of the 26 LITTLE THINGS dwarf galaxies using the high-resolution VLA $\mathrm{H}_{\mathrm{I}}$ data and Spitzer IRAC $3.6 \mu \mathrm{m}$ images as described in Section 2. As mentioned earlier, the simple dynamical structure and dominant circular rotation in the disk of the sample galaxies help to reduce uncertainties stemming from the decomposition of galaxy rotation curves into contributions due to baryonic and non-baryonic matter.

The first step in the mass modeling includes deriving rotation curves of the sample galaxies using the $2 \mathrm{D} \mathrm{H}$ I velocity fields which reflect the total kinematics including both baryons and a DM halo. $\mathrm{H}$ i is mostly distributed in the disk of a galaxy where the circular rotation is dominant and is a useful kinematic tracer for deriving the galaxy rotation curve. This is mainly due to the larger radial extent of $\mathrm{HI}$ in the disk compared to stellar components (e.g., 3-4 times; Sofue \& Rubin 2001). We proceed to derive mass models of the baryons (gas and stars) using $\mathrm{H}_{\mathrm{I}}$ integrated intensity maps and Spitzer IRAC $3.6 \mu \mathrm{m}$ images, and subtract their contribution from the total kinematics. Lastly, we quantify the kinematic residuals in order to examine the DM distribution near the centers of the galaxies. In the following sections, we describe these mass modeling procedures in more detail. 
Table 1

Properties and Tilted-ring Parameters of the LITTLE THINGS Sample Galaxies

\begin{tabular}{|c|c|c|c|c|c|c|c|c|}
\hline $\begin{array}{l}00.0) \\
\text { ") }\end{array}$ & $\begin{array}{c}D \\
(\mathrm{Mpc}) \\
(3)\end{array}$ & $\begin{array}{c}V_{\text {sys }} \\
\left(\mathrm{km} \mathrm{s}^{-1}\right) \\
(4)\end{array}$ & $\begin{array}{l}\langle\text { P. A. }\rangle \\
\left({ }^{\circ}\right) \\
(5)\end{array}$ & $\begin{array}{l}\langle i\rangle \\
\left({ }^{\circ}\right) \\
(6)\end{array}$ & $\begin{array}{c}M_{V} \\
(\mathrm{mag}) \\
(7)\end{array}$ & $\begin{array}{c}12+\log (\mathrm{O} / \mathrm{H}) \\
(8)\end{array}$ & $\begin{array}{c}\log \left(\mathrm{SFR}_{D}^{\mathrm{H} \alpha}\right) \\
\left(M_{\odot} \mathrm{yr}^{-1} \mathrm{kpc}^{-2}\right) \\
(9)\end{array}$ & $\begin{array}{c}\log \left(\mathrm{SFR}_{D}^{\mathrm{FUV}}\right) \\
\left(M_{\odot} \mathrm{yr}^{-1} \mathrm{kpc}^{-2}\right) \\
(10)\end{array}$ \\
\hline 37.5 & 6.1 & $360.8 \pm 1.3$ & $274.1 \pm 5.0$ & $27.9 \pm 0.1$ & -14.7 & $8.1 \pm 0.10$ & $-2.89 \pm 0.01$ & $-2.46 \pm 0.01$ \\
\hline 07.1 & 5.2 & $270.7 \pm 1.3$ & $311.6 \pm 11.9$ & $45.5 \pm 9.0$ & -15.5 & $7.8 \pm 0.20$ & $-2.70 \pm 0.01$ & $-2.40 \pm 0.01$ \\
\hline 24.6 & 3.4 & $156.5 \pm 1.2$ & $175.7 \pm 10.1$ & $49.7 \pm 6.0$ & -16.6 & $7.7 \pm 0.14$ & $-1.67 \pm 0.01$ & $-1.55 \pm 0.01$ \\
\hline 12.9 & 1.3 & $303.5 \pm 1.2$ & $44.5 \pm 13.5$ & $50.0 \pm 0.0$ & -14.1 & $7.5 \pm 0.06$ & $-2.85 \pm 0.01$ & $-2.16 \pm 0.00$ \\
\hline 47.9 & 7.7 & $340.0 \pm 1.0$ & $235.1 \pm 3.9$ & $55.5 \pm 4.8$ & -15.0 & $7.8 \pm 0.04$ & $-1.36 \pm 0.01$ & $-1.00 \pm 0.01$ \\
\hline 19.9 & 6.4 & $589.4 \pm 1.0$ & $287.4 \pm 2.8$ & $51.0 \pm 7.1$ & -15.0 & $8.7 \pm 0.03$ & $-2.85 \pm 0.01$ & $-2.81 \pm 0.01$ \\
\hline 15.9 & 4.9 & $219.4 \pm 1.8$ & $138.0 \pm 3.6$ & $65.0 \pm 0.0$ & -14.9 & $7.8 \pm \ldots$ & $-2.37 \pm 0.01$ & $-2.10 \pm 0.01$ \\
\hline 19.1 & 3.5 & $330.7 \pm 1.0$ & $359.6 \pm 8.4$ & $43.4 \pm 0.1$ & -14.8 & $8.2 \pm 0.09$ & $-2.88 \pm 0.01$ & $-2.62 \pm 0.01$ \\
\hline 09.9 & 3.7 & $372.0 \pm 1.3$ & $226.3 \pm 3.1$ & $68.2 \pm 3.1$ & -14.2 & $7.5 \pm 0.09$ & $-2.50 \pm 0.01$ & $-1.93 \pm 0.01$ \\
\hline 14.1 & 0.7 & $-232.2 \pm 2.2$ & $73.7 \pm 0.0$ & $48.0 \pm 0.0$ & -14.6 & $7.6 \pm 0.05$ & $-2.56 \pm 0.01$ & $-1.99 \pm 0.01$ \\
\hline 10.3 & 3.4 & $-85.4 \pm 5.6$ & $122.5 \pm 1.5$ & $69.1 \pm 0.1$ & -18.2 & $8.2 \pm 0.05$ & $+0.19 \pm 0.01$ & $-0.01 \pm 0.01$ \\
\hline 49.6 & 3.4 & $103.5 \pm 1.4$ & $38.7 \pm 4.2$ & $63.0 \pm 0.8$ & -16.8 & $7.9 \pm 0.01$ & $-1.67 \pm 0.01$ & $-1.66 \pm 0.01$ \\
\hline 44.8 & 4.9 & $235.8 \pm 1.2$ & $292.2 \pm 5.1$ & $22.6 \pm 0.1$ & -17.1 & $8.4 \pm 0.01$ & $-1.66 \pm 0.01$ & $-1.53 \pm 0.01$ \\
\hline 32.4 & 2.6 & $60.5 \pm 1.1$ & $126.1 \pm 3.2$ & $82.5 \pm 0.1$ & -13.6 & $7.9 \pm 0.20$ & $-2.03 \pm 0.01$ & \\
\hline 57.2 & 1.0 & $-122.3 \pm 1.2$ & $174.5 \pm 2.8$ & $74.0 \pm 0.1$ & -14.4 & $7.8 \pm 0.06$ & $-2.77 \pm 0.01$ & $-2.05 \pm 0.01$ \\
\hline 40.4 & 5.9 & $279.3 \pm 2.2$ & $214.5 \pm 2.8$ & $61.2 \pm 4.0$ & -14.6 & $7.9 \pm 0.07$ & $-0.77 \pm 0.01$ & $-1.07 \pm 0.01$ \\
\hline 47.3 & 9.3 & $499.3 \pm 3.9$ & $248.4 \pm 12.5$ & $70.0 \pm 0.0$ & -15.9 & $8.4 \pm 0.08$ & $-1.86 \pm 0.01$ & $-1.55 \pm 0.01$ \\
\hline
\end{tabular}

ved from the tilted-ring analysis in Section 3.1.1; (3): distance as given in Hunter et al. (2012); (4): systemic velocity derived from the tilted-ring analysis in Section 3.1.1; angle (PA) derived from the tilted-ring analysis in Section 3.1.1. PA is the angle measured counter-clockwise from the north direction in the sky to the major axis of the e of the kinematic inclination derived from the tilted-ring analysis in Section 3.1.1; (7): absolute $V$ magnitude as given in Hunter et al. (2012); (8): oxygen abundance taken . (2012). “..." indicates no uncertainty available on the measurements; (9), (10): the $\mathrm{H} \alpha$ and GALEX FUV star formation rates normalized to the area ( $\pi R_{d}^{2}$ where $R_{d}$ is the egreen (2004) and Hunter et al. (2010), respectively. “...” indicates that no measurements are available. 


\subsection{Rotation Curves: Total Kinematics}

\subsubsection{Tilted-ring Fits}

For the derivation of the rotation curves of our sample galaxies, we fit a 2D tilted-ring model which consists of a series of concentric ellipses to the $2 \mathrm{D}$ velocity fields extracted from the $\mathrm{H}_{\mathrm{I}}$ data cubes of the sample galaxies (Rogstad et al. 1974). Each ellipse has its own geometric and kinematic parameters, such as center position (XPOS, YPOS), position angle (PA), inclination (INCL), systemic velocity (VSYS), and rotation velocity (VROT). This socalled "tilted-ring analysis" of 2D velocity fields obtained from $\mathrm{HI}, \mathrm{CO}$ or $\mathrm{H} \alpha$ spectroscopic observations has been widely used for deriving rotation curves of disk-dominated galaxies (e.g., rotation curves of the THINGS galaxies sample; de Blok et al. 2008).

Tilted-ring models, however, only hold for those cases where the velocity field is a reliable representation of the overall kinematics of a galaxy. Non-circular motions caused by starforming activity like stellar winds, $\mathrm{SNe}$, etc., as well as spiral arms, a bar-like or triaxial galaxy potential, galaxy mergers or tidal interactions disturb gas motions in galaxies on small and large scales, resulting in distorted velocity fields.

Low resolution $\mathrm{H}_{\mathrm{I}}$ data usually smooth any features related to small-scale non-circular motions. This is known as beam smearing and tends to yield a velocity gradient along the major axis of a galaxy that is less steep, particularly in the central regions (e.g., de Blok \& McGaugh 1997; Swaters et al. 2000; van den Bosch \& Swaters 2001; McGaugh et al. 2001). Beam smearing is significantly reduced in highresolution data such as THINGS and LITTLE THINGS (see de Blok et al. 2001; McGaugh et al. 2001; Kuzio de Naray et al. 2006).

The LITTLE THINGS sample dwarf galaxies selected in this study, with few exceptions, appear to show no significant largescale kinematic features, such as bars, spiral arms or warps in their H i or Spitzer IRAC $3.6 \mu \mathrm{m}$ images. However, our sample galaxies do suffer from the effect of small-scale turbulent gas motions due to stellar winds and $\mathrm{SNe}$. In general, the derived velocity field in dwarf galaxies is more vulnerable to the impact of stellar activity due to their low gravitational potential (Walter et al. 1998; Walter \& Brinks 2001; see also Bagetakos et al. 2011). Therefore, the extraction of a robust velocity field is essential if one wants to derive the undisturbed underlying kinematics of a galaxy.

In order to correct for small-scale random motions and extract only the component due to circular rotating velocity in a galaxy, we derive the "bulk velocity field" as proposed by Oh et al. (2008, 2011b). Compared with other typical types of velocity fields, such as intensity-weighted mean (IWM), single Gaussian fit and hermite h3, the bulk velocity field has been found to be ideally suited to extracting the underlying bulk rotation of a galaxy in the presence of random non-circular motions. We point out, as an example, how the "kinks" or "wiggles" of the iso-velocity contours of the extracted bulk velocity fields displayed in panel (e) of Figure 8 are weaker than those of the IWM velocity fields (panel b). The bulk velocity field appears to much better represent the overall kinematics of the galaxy and hence the underlying gravitational potential.

Following the standard procedure described in Begeman (1989), we fit tilted-ring models to the bulk velocity fields of the sample galaxies. For this, we use the "rotcur" task in GIPSY $^{27}$ (van der Hulst et al. 1992). The derived rotation curves of the galaxies are presented in the figures of the appendix (e.g., Figure 9). As seen from the scatter in the fits made with all ring parameters free (open circles) in the figures, the extracted bulk velocity fields are not completely free from the effect of small-scale random motions in the galaxies. However, they are relatively insignificant and are averaged out after several iterations as shown in the final rotation curves (solid lines) in the figures.

This is also confirmed in the harmonic analysis of the velocity fields. As described in Schoenmakers et al. (1997), we perform harmonic decompositions of the bulk and IWM velocity fields of the sample galaxies. For this, we use the task "RESWRI" in GIPSY. We expand the velocity fields into sine and cosine terms up to 3rd order (i.e., $c_{m}$ and $s_{m}$ where $m=1,2$ and 3 ) after fixing the center position, PA and INCL with those derived from the tilted-ring analysis. If we allow RESWRI to fit a velocity field with center position, PA and INCL as free parameters, non-circular motions tend to be absorbed into variations in these geometrical parameters, underestimating the amount of non-circular motions. As an example, streaming non-circular motions in a barred galaxy are mainly responsible for the radial motions that are typically reflected in the $s_{1}$ and $s_{3}$ terms (Schoenmakers et al. 1997; Wong et al. 2004; Spekkens \& Sellwood 2007). However, these radial motions can also be modeled by a radial variation of PA, without the need for $s_{1}$ and $s_{3}$ terms. Similarly, other ring parameters can affect the harmonic analysis in the same way if they are kept as free parameters in the fit. To quantify and describe non-circular motions, we calculate the absolute amplitudes $\langle A\rangle$ and the phases of each component decomposed. For the amplitudes, we take the median of $A_{m}(R)$ as described in Schoenmakers et al. (1997) (see also Trachternach et al. 2008),

for $m=1$,

$$
A_{1}(R)=\sqrt{s_{1}(R)^{2}},
$$

for $m>1$,

$$
A_{m}(R)=\sqrt{c_{m}(R)^{2}+s_{m}(R)^{2}},
$$

where $R$ is the galaxy radius.

As shown in the section labeled "Harmonic Analysis" in the appendix, the amplitudes of harmonic terms (e.g., $c_{2}, s_{1}$ and $s_{2}$ which are corrected for inclination) derived from the bulk velocity fields (black dots in the Harmonic Analysis panels) are lower than those derived using the IWM velocity fields over all radii. This shows that the effect of random non-circular motions are largely reduced in the bulk velocity fields. The tilted-ring parameters of the sample galaxies derived using the bulk velocity fields are given in Table 1 . We note that the kinematic center positions given in Table 1 that are derived from the tilted-ring analysis are offset from the optical ( $V$-band, mostly) central isophot used by Hunter et al. (2012).

\subsubsection{Asymmetric Drift Correction}

Pressure support caused by random gas motions in the gaseous disk of a galaxy tends to lower the rotation velocity, which results in an underestimate of the dynamical mass of the

\footnotetext{
27 The Groningen Image Processing System.
} 
galaxy. In general the dynamical effect of the pressure support is higher in the outer region of a galaxy where the gas density is low. In particular, this, so-called "asymmetric drift" is significant in dwarf galaxies whose maximum rotation velocities are comparable to the velocity dispersions in the gas disk. The asymmetric drift correction hence should be made to derive more reliable rotation curves of galaxies where the dynamical support by random motions to its gas disk is significant. This is the case of some of our sample galaxies whose velocity dispersions are comparable to their maximum rotation velocities in the outer region. For the asymmetric drift correction, we follow the method described in Bureau \& Carignan (2002; see also Oh et al. 2011b) as follows.

The asymmetric drift correction $\sigma_{D}$ is given as,

$$
\begin{aligned}
\sigma_{D}^{2} & =-R \sigma^{2} \frac{\partial \ln \left(\rho \sigma^{2}\right)}{\partial R} \\
& =-R \sigma^{2} \frac{\partial \ln \left(\Sigma_{\mathrm{H}} \sigma^{2}\right)}{\partial R},
\end{aligned}
$$

where $\sigma_{D}$ is the asymmetric drift correction, $R$ is the galaxy radius, $\sigma$ is the velocity dispersion, and $\rho$ is the volume density of gas disk. In general, $\rho$ can be approximated as the gas surface density $\Sigma_{\mathrm{H} \text { I }}$ for a gas disk with an exponential distribution in the vertical distribution and a constant scale height $z_{0}$ (i.e., $\left.d\left(\ln \left(z_{0}\right)\right) / d r=0\right)$. Large fluctuations in the derivative in Equation (3) can be smoothed by fitting an analytical function which has three free parameters, $I_{0}$ $\left(M_{\odot} \mathrm{pc}^{-2} \mathrm{~km}^{2} \mathrm{~s}^{-2}\right), R_{0} \quad(\operatorname{arcsec})$, and $\alpha\left(\operatorname{arcsec}^{-1}\right)$ to the numerator as follows,

$$
\Sigma_{\mathrm{H} \mathrm{I}} \sigma^{2}(R)=\frac{I_{0}\left(R_{0}+1\right)}{R_{0}+e^{\alpha R}} .
$$

Lastly, the corrected rotation velocity $V_{\text {cor }}$ is derived by adding the asymmetric drift correction $\sigma_{D}$ to the rotation velocity $V_{\text {rot }}$ derived from tilted-ring fits, quadratically,

$$
V_{\text {cor }}^{2}=V_{\text {rot }}^{2}+\sigma_{D}^{2}
$$

The analytical function given in Equation (4) provides a good fit to most sample galaxies except for DDO 52, IC 10, NGC 3738 and UGC 8508 where a significant degree of radial fluctuation is present in $\Sigma_{\mathrm{H}_{\mathrm{I}}} \sigma^{2}$ at small radii. However, the correction at small radii is insignificant and thus will not affect significantly the final results.

Valenzuela et al. (2007) have shown that the standard asymmetric correction can be underestimated without considering the gas pressure gradients triggered by star formation and feedback. However, the effect of additional thermal pressure gradients in the gas is most likely insignificant for the sample galaxies in this study. The thermal pressure is already included in the observed velocity dispersion used for the asymmetric drift correction as part of its broadening. In addition, as shown in the velocity dispersion map (i.e., moment 2) in the appendix, significant anisotropy in the dispersion is not found in the galaxies. This implies that any separation of turbulent and thermal pressures, with explicit calculation of asymmetries in the turbulent component, would have insignificant effect on the asymmetric drift corrected rotation curves. The asymmetric drift corrected rotation curves of the sample galaxies which are used for the mass modeling are presented in the appendix.

\subsection{Gas Distribution}

The rotation curves derived in Section 3.1 already provide a good approximation of the DM halos' kinematics of the sample galaxies given the dominant contribution of the DM halo to the total kinematics of dwarf galaxies. However, to derive more accurate DM distributions of the galaxies, we construct mass models of their gaseous and stellar components which account for most of the baryons in dwarf galaxies.

We use total integrated $\mathrm{H}_{\mathrm{I}}$ intensity maps (moment 0 ) of the galaxies to derive the mass model of the gaseous component. For consistency with the rotation curves in Section 3.1, we apply the derived tilted-ring models to the $\mathrm{H}_{\mathrm{I}}$ intensity maps, and obtain gas surface density profiles of the galaxies which are scaled up by a factor of 1.4 to take Helium and metals into account. We then convert the gas surface density profiles to the corresponding gas rotation velocities assuming that gas components are mainly distributed in a thin disk. As an example, the derived gas surface density profile and the corresponding rotation velocity of CVnIdwA are shown in the panels $(\mathrm{g})$ and $(\mathrm{h})$ of Figure 10, respectively. Here, we do not correct for the effect of molecular hydrogen $\left(\mathrm{H}_{2}\right)$ since low metallicities in dwarf galaxies can induce only a small fraction of the gaseous component in the form of $\mathrm{H}_{2}$ (e.g., Leroy et al. 2007, 2011; Schruba et al. 2012).

\subsection{Stellar Distribution}

We use Spitzer IRAC $3.6 \mu \mathrm{m}$ images to derive mass models of the stellar components of the galaxies. Compared with optical images, the Spitzer IRAC $3.6 \mu \mathrm{m}$ image is less affected by dust and less sensitive to young stellar populations which usually emit most energy in the optical regime but occupy only a small fraction of the total stellar mass. Instead, the Spitzer IRAC $3.6 \mu \mathrm{m}$ image is useful for tracing old stellar populations that are dominant in late-type dwarf galaxies. This enables us to derive a robust estimate of the stellar mass of our sample galaxies as used for deriving the mass models of the stellar components of THINGS galaxies (de Blok et al. 2008; Oh et al. 2008, 2011b; Trachternach et al. 2008).

Like we did for the gas component, we derive $3.6 \mu \mathrm{m}$ surface brightness profiles of the stellar components of the galaxies by applying the derived tilted-ring parameters to the Spitzer IRAC $3.6 \mu \mathrm{m}$ images as shown in the figures in the appendix (e.g., Figure 10). In general, as discussed in Walter et al. (2008), the Spitzer IRAC $3.6 \mu \mathrm{m}$ image provides a pseudo dust free picture of old stellar populations in galaxies. However, unlike the case of the gas component whose mass can be directly estimated from $\mathrm{H}$ I observations, estimating the stellar mass in galaxies is critically dependent on the assumed stellar mass-to-light ratio $\left(Y_{\star}\right)$ value which usually gives rise to the largest uncertainty when converting the luminosity profile to the mass density profile. In order to derive more reliable $\Upsilon_{\star}$ values in the $3.6 \mu \mathrm{m}$ band, we use an empirical relation between galaxy optical colors and $Y_{\star}^{3.6}$ values based on stellar population synthesis models (Bell \& de Jong 2001; Bruzual \& Charlot 2003) as given in Oh et al. (2008). Hereafter, we call these model $\Upsilon_{\star}^{3.6}$ values. Using the derived $Y_{\star}^{3.6}$ values, we convert the $3.6 \mu \mathrm{m}$ surface brightness profiles of our sample galaxies to stellar 


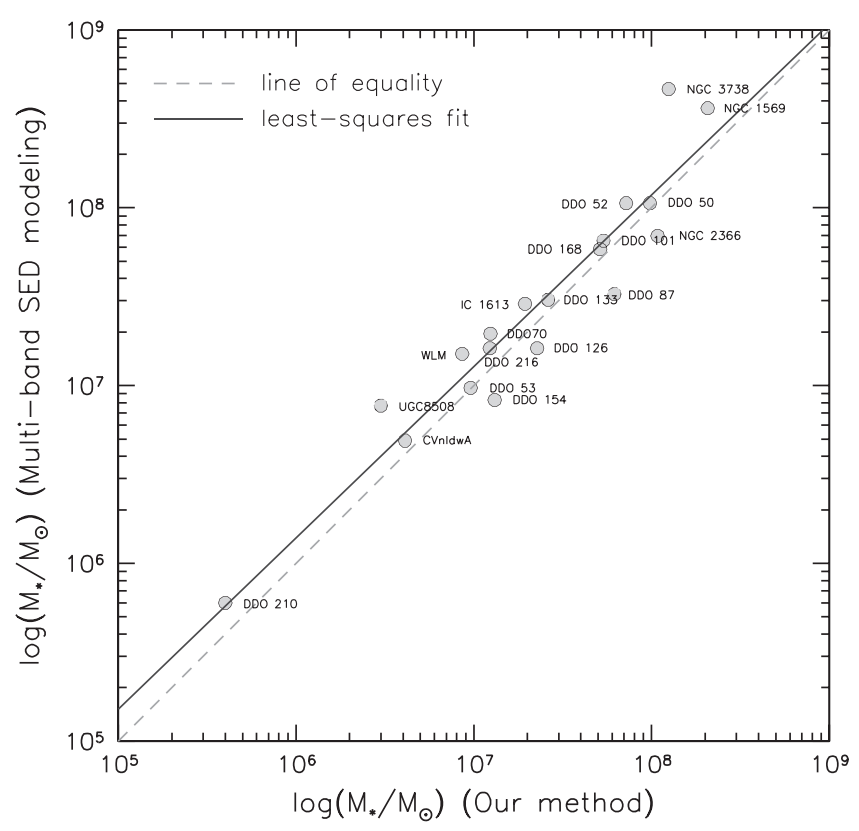

Figure 1. Comparison of the stellar masses derived using our method with those derived using the multi-band spectral energy distribution (SED) fitting technique described in Zhang et al. (2012). The solid and dashed lines indicate a least-squares fit with a slope of 0.96 , and line of equality, respectively. The $1 \sigma$ scatter of the fit to the data is 0.20 dex.

surface density profiles (see the figures in the appendix, e.g., Figure 10).

As discussed in Oh et al. (2011a), the kinematic method combined with the model $Y_{\star}^{3.6}$ values has been found to be reliable for estimating stellar masses of late-type dwarf galaxies based on a comparison of the derived stellar masses of the simulated dwarf galaxies to the input ones. This robustly supports the veracity of the methodology used for measuring the stellar masses of our sample galaxies. In addition, we also refer to the stellar masses of the sample galaxies derived using a spectral energy distribution (SED) fitting technique (Zhang et al. 2012). As shown in Figure 1, the stellar masses derived using these two independent methods show good agreement within the scatter.

Following Oh et al. (2011b), we calculate the corresponding rotation velocities of the stellar components of the sample galaxies from the derived surface density profiles. For this, we assume a vertical $\operatorname{sech}^{2}(z)$ scale height distribution of stars with a ratio of $h / z_{0}=5$ where $h$ and $z_{0}$ are the radial scale length and the vertical scale height of stellar disk in the $3.6 \mu \mathrm{m}$ surface brightness profiles, respectively (van der Kruit \& Searle 1981; Kregel et al. 2002). Although it may overestimate the rotation velocities of the stellar components of galaxies with a fatter stellar disk, this is a valid assumption for most disk-dominated dwarf galaxies like our sample galaxies. The derived rotation velocities of the stellar components of the sample galaxies are shown in the figures in the appendix (e.g., panel (d) of Figure 10), and the stellar masses estimated in this paper are given in Table 2.

The mean ratio of the masses between the gas and stellar components of the sample galaxies, $\left\langle M_{\text {gas }} / M_{\text {star }}^{\mathrm{KIN}}\right\rangle$ is $\sim 5.6$ which is consistent with $\left\langle M_{\text {gas }} / M_{\text {star }}^{\text {SED }}\right\rangle \sim 7.2$ (where $M_{\text {star }}^{\text {SED }}$ is derived using a SED fitting technique) of 34 LITTLE THINGS sample galaxies in Zhang et al. (2012). This indicates that the majority of the baryons in our sample galaxies is in the form of gaseous components. As discussed earlier, given that the mass of gaseous components in galaxies can be reliably measured from $\mathrm{H}$ I observations without any critical assumption, the mass models of the baryons derived in this study are likely to provide a good description of the distribution of baryons in the galaxies. Therefore, any remaining uncertainties in the mass models of baryons are most likely to be insignificant and thus will not affect significantly the final mass models of the DM halos of our sample galaxies.

\subsection{Disk-halo Decomposition}

In this section, we decompose the total kinematics of the sample galaxies into the dynamical contributions of the baryonic disks and DM halos by disentangling the mass models of baryons from the total rotation curves. For this, we subtract in quadrature the rotation velocities of the gas and stellar components from the total rotation velocities, and obtain implied rotation curves for the DM halos as shown in the figures in the appendix (e.g., see the left-lower panel of Figure 10). For a quantitative analysis of the DM distribution in the galaxies, we fit CDM (Navarro et al. 1996b, 1997; hereafter NFW) and spherical pseudo-isothermal halo models (e.g., Begeman et al. 1991), the two representative cusp- and corelike halo models, to the DM halo rotation curve, respectively. An Einasto profile which is a Sérsic function in the context of CDM halos has been found to provide an equal or better fit to the halos in pure DM simulations compared to an NFW profile Navarro et al. (2004b). In this work, we use NFW profiles to quantify the DM halos of the sample galaxies for consistency with the previous DM mass modeling of THINGS and simulated dwarf galaxies in $\mathrm{Oh}$ et al. (2011a, 2011b). A relative comparison of the two halo models in terms of fit quality enables us to examine which model best describes the DM component of the sample galaxies, especially toward the centers of the galaxies.

\subsubsection{Cusp-like Halo Model}

The cosmologically motivated NFW halo model, the socalled "universal density profile" which describes the cusp-like radial DM distribution found in DM-only $\Lambda$ CDM simulations is given as,

$$
\rho_{\mathrm{NFW}}(R)=\frac{\rho_{i}}{\left(R / R_{S}\right)\left(1+R / R_{s}\right)^{2}},
$$

where $\rho_{i}$ is correlated with the mean density of the universe at the time of the collapse of the halo and $R_{s}$ is the characteristic radius of the DM halo (Navarro et al. 1996b). This profile has been widely adopted to account for the DM distribution which steeply increases toward the centers of the halos in the simulations. This, a so-called cusp feature, can be well approximated by a power law, $\rho \sim R^{\alpha}$ with $\alpha \sim-1.0$ near the central region of the halos, giving a DM halo rotation velocity as follows,

$$
V_{\mathrm{NFW}}(R)=V_{200} \sqrt{\frac{\ln (1+c x)-c x /(1+c x)}{x[\ln (1+c)-c /(1+c)]}},
$$

where $c$ is the concentration parameter defined as $R_{200} / R_{s} . V_{200}$ is the rotation velocity at a radius $R_{200}$ where the mass density contrast with the critical density of the universe exceeds 200 , and $x$ is defined as $R / R_{200}$ (Navarro et al. 1996b). In particular, 
Table 2

Mass Modeling Results of the LITTLE THINGS Sample Galaxies

\begin{tabular}{|c|c|c|c|c|c|c|c|c|c|c|c|c|}
\hline $\mathrm{R}_{\max } \mathrm{HI}_{\text {beam }}^{-1}$ & $\begin{array}{c}z_{0} \\
(\mathrm{kpc}) \\
(6)\end{array}$ & (7) & $\begin{array}{c}V_{200} \\
\left(\mathrm{~km} \mathrm{~s}^{-1}\right) \\
(8)\end{array}$ & $\begin{array}{c}R_{\mathrm{C}} \\
(\mathrm{kpc}) \\
(9)\end{array}$ & $\begin{array}{c}\rho_{0} \\
\left(10^{-3} M_{\odot} \mathrm{pc}^{-3}\right) \\
(10)\end{array}$ & $\begin{array}{l}\alpha_{\min } \\
(11)\end{array}$ & $\begin{array}{l}\alpha_{Y_{\star}^{3.6}} \\
(12)\end{array}$ & $\begin{array}{c}M_{\mathrm{gas}} \\
\left(10^{7} M_{\odot}\right) \\
(13)\end{array}$ & $\begin{array}{c}M_{\text {star }}^{\mathrm{KIN}} \\
\left(10^{7} M_{\odot}\right) \\
(14)\end{array}$ & $\begin{array}{c}M_{\mathrm{star}}^{\mathrm{SED}} \\
\left(10^{7} M_{\odot}\right) \\
(15)\end{array}$ & $\begin{array}{c}\log \left(M_{\text {dyn }}\right) \\
\left(M_{\odot}\right) \\
(16)\end{array}$ & $\begin{array}{c}\log \left(M_{200}\right) \\
\left(M_{\odot}\right) \\
(17)\end{array}$ \\
\hline 13.5 & 0.24 & $-0.4(11.0)$ & $478.5(16.1 \pm 1.6)$ & $2.01 \pm 0.52$ & $8.19 \pm 1.62$ & $-1.25 \pm 0.21$ & $+0.03 \pm 0.27$ & 2.91 & 0.41 & 0.49 & 8.529 & 9.138 \\
\hline 13.7 & $\ldots$ & $5.6 \pm 2.6(10.2)$ & $39.8 \pm 14.9(31.4 \pm 1.8)$ & $0.94 \pm 0.18$ & $33.22 \pm 8.78$ & $-0.15 \pm 0.13$ & $-0.25^{\mathrm{a}} \pm 0.16$ & 23.26 & $\ldots$ & $\ldots$ & 9.159 & 10.317 \\
\hline 15.7 & $\ldots$ & $18.7 \pm 3.5(9.0)$ & $61.2 \pm 9.7(136.7 \pm 6.1)$ & $0.51 \pm 0.04$ & $517.82 \pm 65.46$ & $-0.41 \pm 0.01$ & $-0.42^{\mathrm{a}} \pm 0.02$ & 22.08 & $\ldots$ & $\ldots$ & 9.609 & 11.925 \\
\hline 29.3 & $\ldots$ & $-0.4(9.2)$ & $1359.0(51.3 \pm 2.5)$ & $3.72 \pm 0.48$ & $12.15 \pm 1.49$ & $-1.24 \pm 0.20$ & $-1.25^{\mathrm{a}} \pm 0.22$ & 46.80 & $\ldots$ & $\ldots$ & 9.930 & 10.648 \\
\hline 84.4 & 0.28 & $22.2(10.3)$ & $15.4(28.8 \pm 0.2)$ & $0.15 \pm 0.08$ & $379.64 \pm 381.14$ & $-0.41 \pm 0.41$ & $+0.10 \pm 0.41$ & 132.52 & 9.79 & 10.60 & 9.463 & 9.895 \\
\hline 15.9 & 0.26 & $6.1 \pm 1.1(9.4)$ & $71.9 \pm 11.8(57.8 \pm 1.3)$ & $1.33 \pm 0.07$ & $48.81 \pm 3.63$ & $-0.55 \pm 0.04$ & $-0.49 \pm 0.02$ & 33.43 & 7.20 & 5.31 & 9.664 & 10.803 \\
\hline 13.1 & 0.14 & $0.0 \pm 4.4(10.4)$ & $425.8 \pm 2531.9(27.8 \pm 3.2)$ & $2.22 \pm 1.95$ & $25.10 \pm 5.63$ & $-0.27 \pm 0.48$ & $+0.14 \pm 0.80$ & 7.00 & 0.96 & 0.97 & 8.567 & 9.849 \\
\hline 22.9 & 0.11 & $0.0 \pm 10.2(10.3)$ & $866.1 \pm 11717.4(38.6 \pm 1.8)$ & $0.51 \pm 0.11$ & $119.95 \pm 34.69$ & $-0.43 \pm 0.02$ & $-0.48 \pm 0.03$ & 3.80 & 1.24 & 1.96 & 8.768 & 10.277 \\
\hline 26.1 & 0.48 & $0.0 \pm 0.2(9.5)$ & $511.4 \pm 143.8(40.7 \pm 1.6)$ & $2.46 \pm 0.11$ & $13.91 \pm 0.77$ & $-0.01 \pm 0.44$ & $-0.01 \pm 0.47$ & 29.12 & 6.18 & 3.27 & 9.734 & 10.346 \\
\hline 7.5 & 0.13 & $25.0 \pm 1.3(9.4)$ & $43.9 \pm 1(115.7 \pm 6.4)$ & $0.32 \pm 0.01$ & $849.14 \pm 77.23$ & $-1.00 \pm 0.15$ & $-1.02 \pm 0.12$ & 3.48 & 5.79 & 6.54 & 9.279 & 510.18 \\
\hline 24.3 & 0.23 & $-0.2 \pm 44.4(10.1)$ & $626.6 \pm 48442.1(31.4 \pm 1.2)$ & $1.33 \pm 0.10$ & $21.59 \pm 2.00$ & $-0.41 \pm 0.15$ & $-0.39 \pm 0.15$ & 16.36 & 2.27 & 1.62 & 9.182 & 10.008 \\
\hline 16.5 & 0.22 & $4.9 \pm 2.3(9.8)$ & $74.5 \pm 36.7(46.6 \pm 1.6)$ & $0.83 \pm 0.06$ & $73.69 \pm 7.61$ & $-0.11 \pm 0.14$ & $-0.11 \pm 0.15$ & 12.85 & 2.62 & 3.04 & 9.261 & 10.522 \\
\hline 51.3 & 0.14 & $6.4 \pm 0.5(9.8)$ & $48.7 \pm 2.9(41.1 \pm 0.6)$ & $0.95 \pm 0.03$ & $53.21 \pm 3.19$ & $-0.39 \pm 0.11$ & $-0.41 \pm 0.13$ & 35.27 & 1.31 & 0.83 & 9.614 & 10.359 \\
\hline 19.2 & 0.22 & $-0.3 \pm 1171.6(9.2)$ & $2290.8 \pm \ldots(63.9 \pm 4.0)$ & $2.81 \pm 0.83$ & $39.81 \pm 6.37$ & $-0.28 \pm 0.28$ & $+0.97 \pm 0.18$ & 25.94 & 5.13 & 5.85 & 9.520 & 10.934 \\
\hline 6.0 & 0.10 & $0.0 \pm 202.3(12.2)$ & $339.4 \pm \ldots(12.4 \pm 1.0)$ & $0.20 \pm 0.05$ & $116.43 \pm 23.05$ & $-0.30 \pm 0.07$ & $-0.70 \pm 0.02$ & 0.14 & 0.04 & 0.06 & 6.964 & 8.797 \\
\hline 13.0 & 0.27 & $13.2 \pm 2.7(11.7)$ & $8.8 \pm 1.0(14.7 \pm 0.4)$ & $0.15 \pm 0.03$ & $127.02 \pm 43.35$ & $-0.17 \pm 0.47$ & $-0.30 \pm 0.62$ & 0.49 & 1.60 & 1.51 & 7.807 & 9.019 \\
\hline 7.0 & $\ldots$ & $10.8 \pm 4.5(10.4)$ & $26.2 \pm 7.1(29.6 \pm 2.1)$ & $0.55 \pm 0.20$ & $74.24 \pm 40.17$ & $-0.66^{\mathrm{a}} \pm 0.14$ & $-0.68^{\mathrm{a}} \pm 0.18$ & 4.37 & $\ldots$ & $\ldots$ & 9.003 & 9.931 \\
\hline 27.0 & 0.07 & $0.2 \pm 951.3(18.6)$ & $2077.1 \pm \ldots(36.8 \pm 2.2)$ & $0.27 \pm 0.12$ & $190.4 \pm 76.4$ & $-1.19 \pm 0.01$ & $-0.25 \pm 0.32$ & 1.65 & 11.81 & $\ldots$ & 8.213 & 10.215 \\
\hline 103.8 & 0.15 & $5.0 \pm \ldots(11.2)$ & $5.4 \pm \ldots(12.5 \pm 0.4)$ & $0.20 \pm 0.04$ & $19.25 \pm 3.45$ & $+1.16 \pm 1.22$ & $-0.10 \pm 0.92$ & 5.93 & 1.94 & 2.88 & 8.448 & 8.808 \\
\hline 31.5 & 0.19 & $-0.3 \pm 179.2(9.8)$ & $736.6 \pm \ldots(40.9 \pm 2.2)$ & $2.71 \pm 0.81$ & $15.23 \pm 2.45$ & $-0.77 \pm 0.17$ & $-0.23 \pm 0.67$ & 20.24 & 20.69 & 36.29 & 9.193 & 10.352 \\
\hline 70.7 & 0.29 & $5.2 \pm 0.6(9.4)$ & $66.0 \pm 6.1(52.6 \pm 0.8)$ & $1.21 \pm 0.04$ & $43.89 \pm 2.51$ & $-0.52 \pm 0.13$ & $-0.53 \pm 0.12$ & 108.24 & 10.81 & 6.94 & 9.841 & 10.680 \\
\hline 11.7 & 0.12 & $12.4 \pm 15.4(8.1)$ & $310.4 \pm 536.2(598.2 \pm 30.4)$ & $0.45 \pm 0.04$ & $2132.36 \pm 277.75$ & $-0.42 \pm 0.03$ & $-0.44 \pm 0.02$ & 12.58 & 12.48 & 46.62 & 9.838 & 13.848 \\
\hline 25.0 & 0.09 & $7.8 \pm 1.4(10.0)$ & $54.7 \pm 7.4(44.2 \pm 2.2)$ & $1.95 \pm 0.21$ & $45.29 \pm 2.38$ & $-0.31 \pm 0.17$ & $-0.38 \pm 0.16$ & 1.19 & 0.30 & 0.77 & 8.913 & 10.453 \\
\hline 82.9 & 0.17 & $3.0 \pm 0.8(10.2)$ & $90.8 \pm 26.1(33.3 \pm 0.5)$ & $0.74 \pm 0.01$ & $57.46 \pm 1.57$ & $+0.03 \pm 0.01$ & $-0.02 \pm 0.01$ & 7.96 & 1.23 & 1.62 & 9.002 & 10.085 \\
\hline 25.7 & $\ldots$ & $19.5 \pm 1.9(10.4)$ & $23.4 \pm 0.9(32.7 \pm 0.9)$ & $0.23 \pm 0.04$ & $414.68 \pm 137.20$ & $-0.50 \pm 0.12$ & $-0.50^{\mathrm{a}} \pm 0.12$ & 9.35 & $\ldots$ & 1.43 & 9.156 & 10.061 \\
\hline 10.0 & 0.13 & $-0.4 \pm 1503.4(9.7)$ & $1649.2 \pm \ldots(47.9 \pm 3.5)$ & $8.40 \pm 12.17$ & $16.99 \pm 2.78$ & $-0.48 \pm 0.06$ & $-0.50 \pm 0.01$ & 11.16 & 5.81 & $\ldots$ & 9.308 & 10.558 \\
\hline
\end{tabular}

It of the rotation curve is measured; (2): the radius where the logarithmic slope of the total rotation curve (DM + baryons) $d \log V / d \log R=0.3$. See Section 4.1; (3): the at $R_{\max }$; (4): the rotation velocity of the best fitted pseudo-isothermal halo model at $R_{\max }$; (5): the ratio of $R_{\max }$ to $\mathrm{H}$ I beam size; (6): the vertical scale height of the stellar otation velocity $V_{200}$ for a NFW halo model (Navarro et al. 1995, 1996b); $c$ values in brackets are derived using an empirical relationship between $c-V_{200}$ as given by $V_{200}$ values are fitted after fixing $c$ to the ones in brackets assuming a "minimum disk." See Section 3.4 for a detailed description. "..." indicates that the uncertainty is core density of a pseudo-isothermal halo model (Begeman et al. 1991); (11): the logarithmic inner slope $\alpha_{\min }$ of the total matter (DM halo + baryons) density profiles ic inner slope $\alpha_{Y_{x}^{3} .6}$ of the DM density profiles measured in Section 4.2. (13): The gas mass derived in Section 3.2; (14): the stellar mass derived from the kinematic analysis using a spectral energy distribution (SED) fitting technique in Zhang et al. (2012); (16): the dynamical mass measured using $V_{\text {ISO }}\left(R_{\max }\right)$ and $R_{\max } ;(17)$ : CDM halo mass column (5) and using Equation 3 given in Oh et al. (2011a). See Section 3.4 for more details.

omponent is included in the DM density profile as no Spitzer $3.6 \mu \mathrm{m}$ image is available for this galaxy. 
the concentration parameter $c$ is useful for quantifying the degree of DM concentration in galaxies. The measurement of the value of $c$ in nearby galaxies provides an observational constraint on the central cusps predicted from $\Lambda \mathrm{CDM}$ simulations (McGaugh et al. 2007; see also de Blok et al. 2003).

\subsubsection{Core-like Halo Model}

As an alternative way to describe the DM distribution in a galaxy observationally motivated, spherical pseudo-isothermal halo models with a central constant-density core have been used in studies of galaxy rotation curves. The form of this corelike halo model is given as follows:

$$
\rho_{\mathrm{ISO}}(R)=\frac{\rho_{0}}{1+\left(R / R_{C}\right)^{2}},
$$

where $\rho_{0}$ and $R_{C}$ are the core density and core radius of a halo, respectively. This halo model is employed to describe the mass distribution of a DM halo with a sizeable constant density core $\left(\rho \propto \rho_{0}\right)$. Similarly, the corresponding rotation velocity to the pseudo-isothermal halo potential is given by,

$$
V_{\mathrm{ISO}}(R)=\sqrt{4 \pi G \rho_{0} R_{C}^{2}\left[1-\frac{R_{C}}{R} \operatorname{atan}\left(\frac{R}{R_{C}}\right)\right]} .
$$

In order to quantify the DM distribution in the sample galaxies, we fit the model rotation velocities of the two halo models as given in Equations (7) and (9) to the kinematic DM signature of the galaxies. As shown in the figures in the appendix (e.g., the left-lower panel of Figure 10), the core-like pseudo-isothermal halo models are mostly preferred over the cusp-like NFW models in terms of the quality of the fit (i.e., based on reduced $\chi^{2}$ values). The fitted parameters of the halo models are presented in Table 2.

As given in Table 2, we derive the dynamical masses $\left(M_{\text {dyn }}\right)$ of the sample galaxies using $V_{\mathrm{ISO}}\left(R_{\max }\right)$ and $R_{\max }$ at which the outermost part of the rotation curve is measured. Given that rotation curves at the adopted $R_{\max }$ are mostly rising in our sample galaxies, the derived dynamical mass, $M_{\text {dyn }}$ with $V_{\mathrm{ISO}}\left(R_{\max }\right)$ and $R_{\max }$ gives a lower limit of the halo mass. For reference, we also estimate the halo masses, $M_{200}$, of our sample galaxies assuming an NFW halo model. However, the fitted values of $c$ are unphysical (i.e., negative or close to zero) for a large fraction (11/26 galaxies) of the sample galaxies. We therefore derive a $c$ value using an empirical relationship between $c-V_{200}$ from the WMAP $P^{28}$ observations in McGaugh et al. (2007) by substituting $V_{200}$ with the $V_{\text {ISO }}\left(R_{\max }\right)$ adopted. We then fit the NFW halo model after fixing $c$ and leaving only $V_{200}$ as a free parameter, assuming a minimum disk (where the rotation curve is attributed to the DM halo only and the dynamical contribution of baryons is ignored). These $c$ and $V_{200}$ values are given in brackets in Table 2. Lastly, we derive the resulting halo mass, $M_{200}$, with the newly estimated $V_{200}$ using Equation (3) in Oh et al. (2011a). As presented in Table 2, $M_{200}$ values of the sample galaxies are larger than $M_{\text {dyn }}$ values, which implies that our observations most likely do not reach the flat part of the rotation curves. This is consistent with the fact that the rotation curves of most sample galaxies are still rising at the last measured points.

\footnotetext{
${ }^{28}$ The WMAP (Spergel et al. 2003, 2007).
}

Except in those few cases, such as DDO 70, DDO 101, DDO 154, DDO 210, DDO 216, and Haro 36 where CDM NFW halo models provide comparable fits to the DM rotation curves, the fitted values of the NFW halo parameters are unphysical (i.e., negative concentration parameter $c$, unphysically large values of $\left.V_{200}\right)$. This is consistent with results previously found in other nearby dwarf and LSB galaxies (e.g., de Blok \& Bosma 2002; Kuzio de Naray et al. 2008; van Eymeren et al. 2009; Oh et al. 2011b). The slowly increasing DM rotation curves in the inner region of the sample galaxies reflect a halo whose gravitational potential is not deep enough to sustain the power-law DM density cusps that are as steep as $\rho \propto R^{-1.0}$. Hence, the cosmologically motivated cusp-like halo models are not able to adequately describe the observed solidbody rotation curves of our sample dwarf galaxies.

It may be argued that those galaxies that are equally well fitted by the two halo models indicate the possibility of a kinematic signature of central cusps in dwarf galaxies being consistent with $\Lambda \mathrm{CDM}$ simulations. Despite the high-resolution of LITTLE THINGS VLA H I observations, it is, however, most likely that the central regions of the galaxies are not fully sampled with a sufficient number of independent synthesized beams needed for accurately distinguishing the inner steepness of the two halo models. As quantified in the parameter, $R_{\max } \mathrm{H}_{\mathrm{I}}$ -1 beam in Table 2, this is mainly due to the small size of the rotating disk (e.g., DDO 210) or the relatively large distance (e.g., Haro 36). Given that the difference between the cuspand core-like halo models is the most prominent in the central regions of galaxies, higher sampling of the inner regions of the galaxies is required before making a firmer conclusion on the signature of the potential central cusps. We will discuss this matter in a more quantitative way in the following section.

\section{DM DISTRIBUTION}

In this section, we compare the inner shape of rotation curves and DM density profiles of the sample galaxies with those of simulated dwarf galaxies from $N$-body+SPH $\Lambda$ CDM simulations in order to examine their DM distributions near the centers. These comparisons between observations and simulations allow us to estimate the degree of cuspiness of the central DM distribution in a qualitative way. In addition, we also measure the inner DM density slopes of the galaxies to make a more quantitative comparison to the simulations.

\subsection{Rotation Curve Shape}

The characteristic shape of the steeply rising rotation curve inherent in the cusp-like DM distribution near the centers of simulated dwarf galaxies based on the $\Lambda \mathrm{CDM}$ paradigm can be used for a qualitative test of the simulations (Hayashi \& Navarro 2006; see also $\mathrm{Oh}$ et al. 2011b, 2011a). This qualitative test is particularly useful in that a direct comparison between the observed and predicted DM rotation curves can be made without any additional assumption on the shape of the DM halo (e.g., a spherical or triaxial halo potential) which is needed for converting rotation curves to the corresponding density profiles, and the associated additional uncertainties this might introduce.

This is done in the left panel of Figures 2-4 where we scale the rotation curves of both our sample galaxies and $\Lambda$ CDM NFW halos with respect to the rotation velocity $V_{0.3}$ at a radius $R_{0.3}$ which is where the logarithmic slope of the 


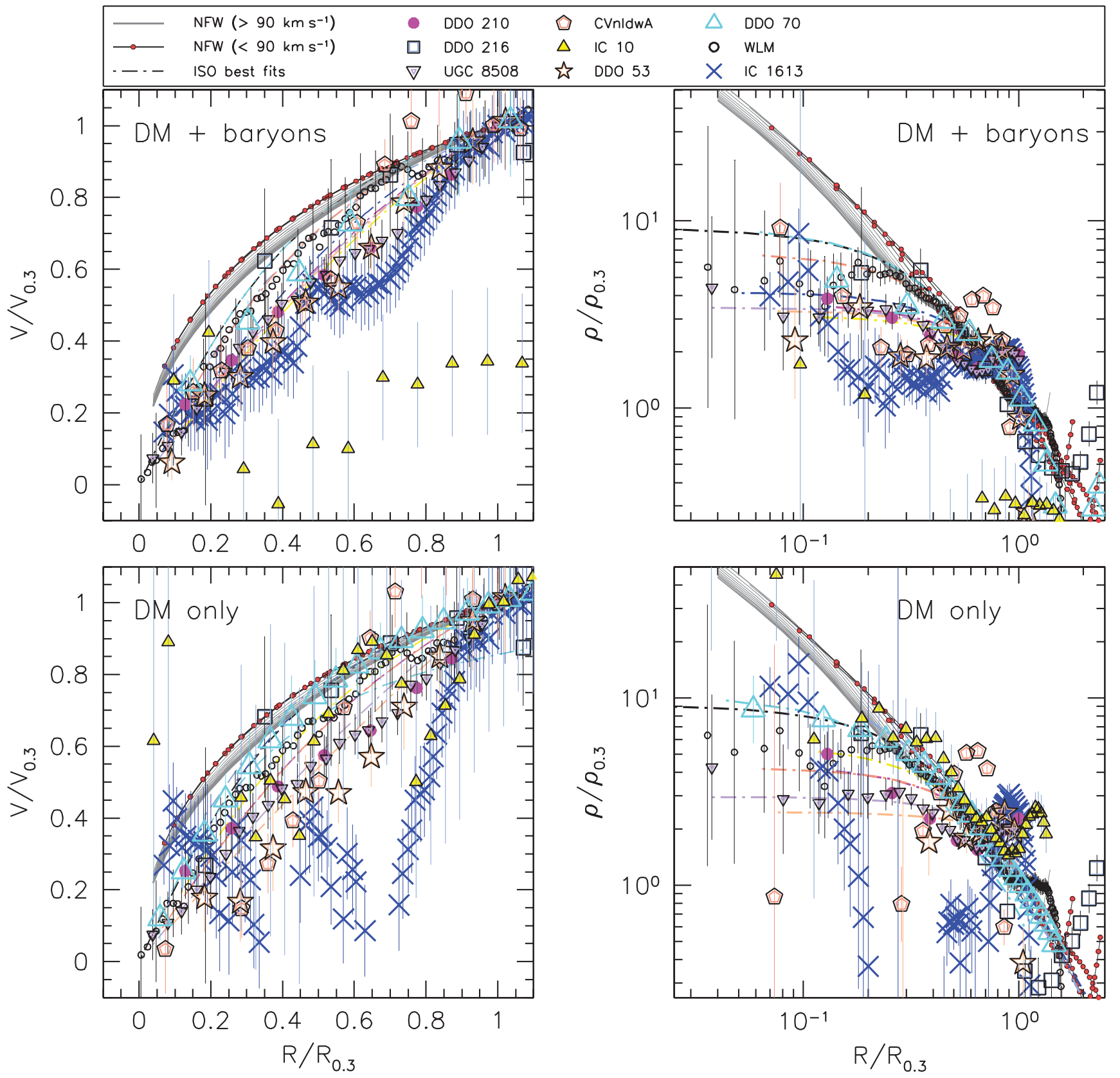

Figure 2. Left panels: the rotation curves of the first nine galaxies of the 26 LITTLE THINGS (in dynamical mass order) which are all scaled with respect to the rotation velocity $V_{03}$ at $R_{03}$ where the logarithmic slope of the rotation curve is $d \log V / d \log R=0.3$ as described in Hayashi \& Navarro (2006). The upper (DM + baryons) and lower (DM only) panels show the ones including and excluding the dynamical contribution by baryons, respectively. The gray solid and black solid lines with small dots indicate the CDM NFW dark matter rotation curves with $V_{200}$ which is $>90 \mathrm{~km} \mathrm{~s}^{-1}$ and $<90 \mathrm{~km} \mathrm{~s}^{-1}$, respectively. The dashed lines (denoted as $I S O$ ) show the best fitted pseudo-isothermal halo models to the galaxies. Right panels: the corresponding dark matter density profiles derived using the scaled rotation curves in the left panels. The gray $\left(V_{200}>90 \mathrm{~km} \mathrm{~s}^{-1}\right)$ and black solid lines with small dots $\left(V_{200}<90 \mathrm{~km} \mathrm{~s}^{-1}\right)$ represent the CDM NFW models with the inner density slope $\alpha \sim-1.0$. The dashed lines indicate the best fitted pseudo-isothermal halo models with $\alpha \sim 0.0$. See Section 4 for more details.

The data used to create this figure are available.

curve is $d \log V / d \log R=0.3$ (see Hayashi \& Navarro 2006). This enables us not only to make a relative comparison of the rotation curves between observations and simulations but also to accentuate the inner rotation curve shape which is sensitive to the degree of central DM concentration.

All the scaled (DM only) rotation curves of the 21 LITTLE THINGS sample galaxies for which Spitzer $3.6 \mu \mathrm{m}$ image is available are overplotted in the upper-left panel of Figure 5. We also overplot the median values of the rotation curves in each $0.1 \mathrm{R} / \mathrm{R}_{0.3}$ bin. In the lower-left panel of Figure 5 , we also overplot the scaled rotation curves of seven dwarf galaxies from THINGS (three of them are also in LITTLE THINGS) as well as the two simulated dwarf galaxies presented in Governato et al. (2010) which all show a linear increase in their inner regions to the median values of the LITTLE THINGS rotation curves. In particular, the two simulated dwarf galaxies were affected by baryonic feedback processes (mainly repeated gas outflows driven by SN explosions) in such a way that the central cusps predicted from DM-only simulations are flattened (Governato et al. 2010). The flattened DM 


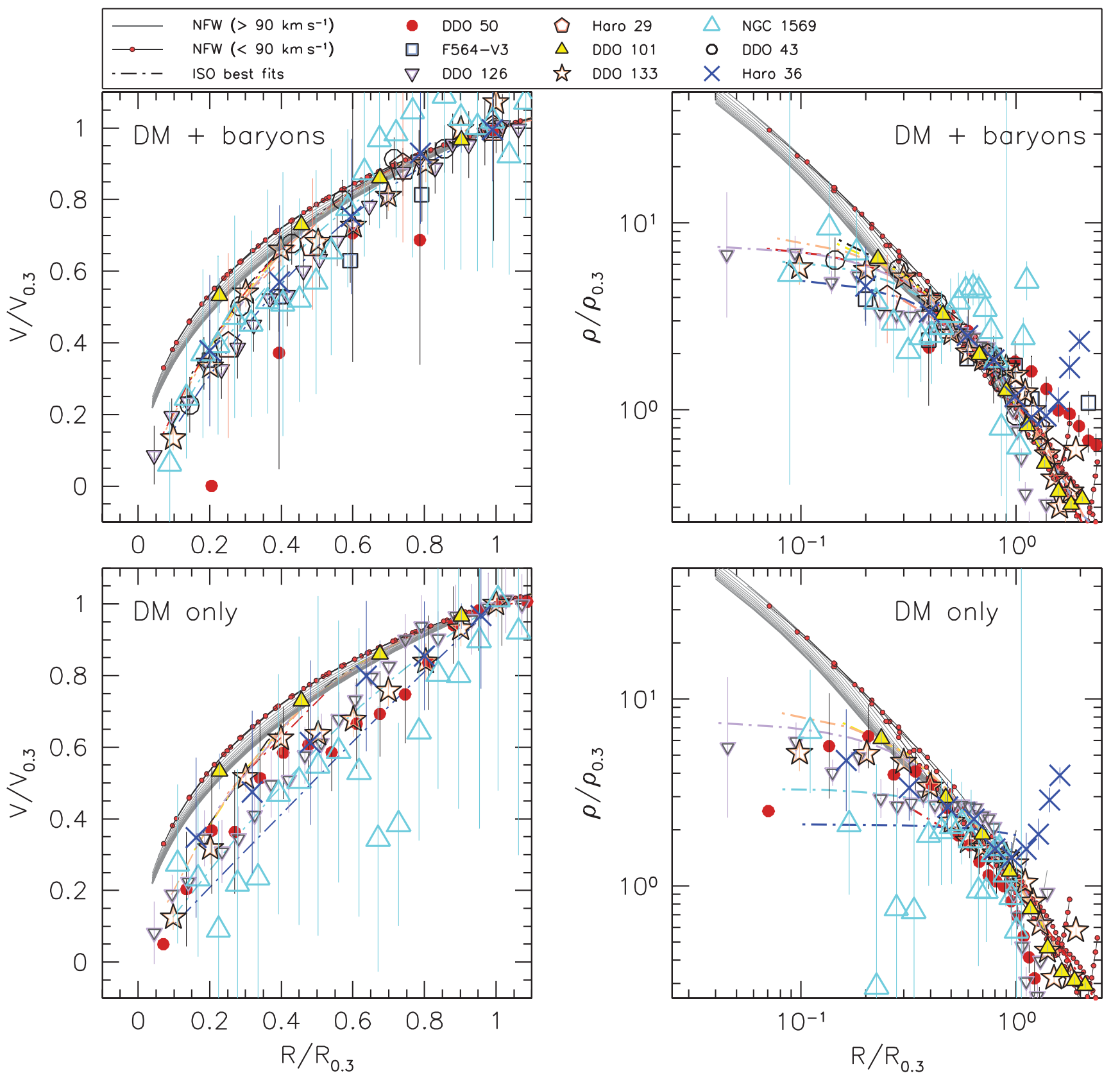

Figure 3. Left panels: the rotation curves of the other nine galaxies of the 26 LITTLE THINGS (in dynamical mass order) which are all scaled with respect to the rotation velocity $V_{0.3}$ at $R_{0.3}$ where the logarithmic slope of the rotation curve is $d \log V / d \log R=0.3$ as described in Hayashi \& Navarro (2006). The upper (DM + baryons) and lower (DM only) panels show the ones including and excluding the dynamical contribution by baryons, respectively. The gray solid and black solid lines with small dots indicate the CDM NFW dark matter rotation curves with $V_{200}$ which is $>90 \mathrm{~km} \mathrm{~s}^{-1}$ and $<90 \mathrm{~km} \mathrm{~s}{ }^{-1}$, respectively. The dashed lines (denoted as $I S O$ ) show the best fitted pseudo-isothermal halo models to the galaxies. Right panels: the corresponding dark matter density profiles derived using the scaled rotation curves in the left panels. The gray $\left(V_{200}>90 \mathrm{~km} \mathrm{~s}^{-1}\right)$ and black solid lines with small dots $\left(V_{200}<90 \mathrm{~km} \mathrm{~s}{ }^{-1}\right)$ represent the CDM NFW models with the inner density slope $\alpha \sim-1.0$. The dashed lines indicate the best fitted pseudo-isothermal halo models with $\alpha \sim 0.0$. See Section 4 for more details (continued).

The data used to create this figure are available.

distribution results in slowly increasing rotation curves in the inner region of the simulated galaxies (see Oh et al. 2011a for further discussion).

In line with the results in Oh et al. (2011a), the inner shape of the scaled rotation curves of the LITTLE THINGS sample galaxies falls mostly below that of the DM-only NFW halo models, indicating a shallower DM distribution near the centers. As shown in the lower-left panel of Figures 2-4, the discrepancy with NFW models becomes more pronounced when comparing these models to DM rotation curves derived after subtracting the contribution from baryons from the total kinematics for each of the sample galaxies. Instead, they are more in line with those of both the THINGS dwarf galaxies and simulations (DG1 and DG2) where the effect of baryonic feedback processes is included. The linearly (or less steeply) rising rotation curves in the inner region of the galaxies indicate a nearly constant or shallower mass distribution toward the centers as found in the majority of nearby dwarf galaxies. The LITTLE THINGS sample galaxies give no clear indication of 


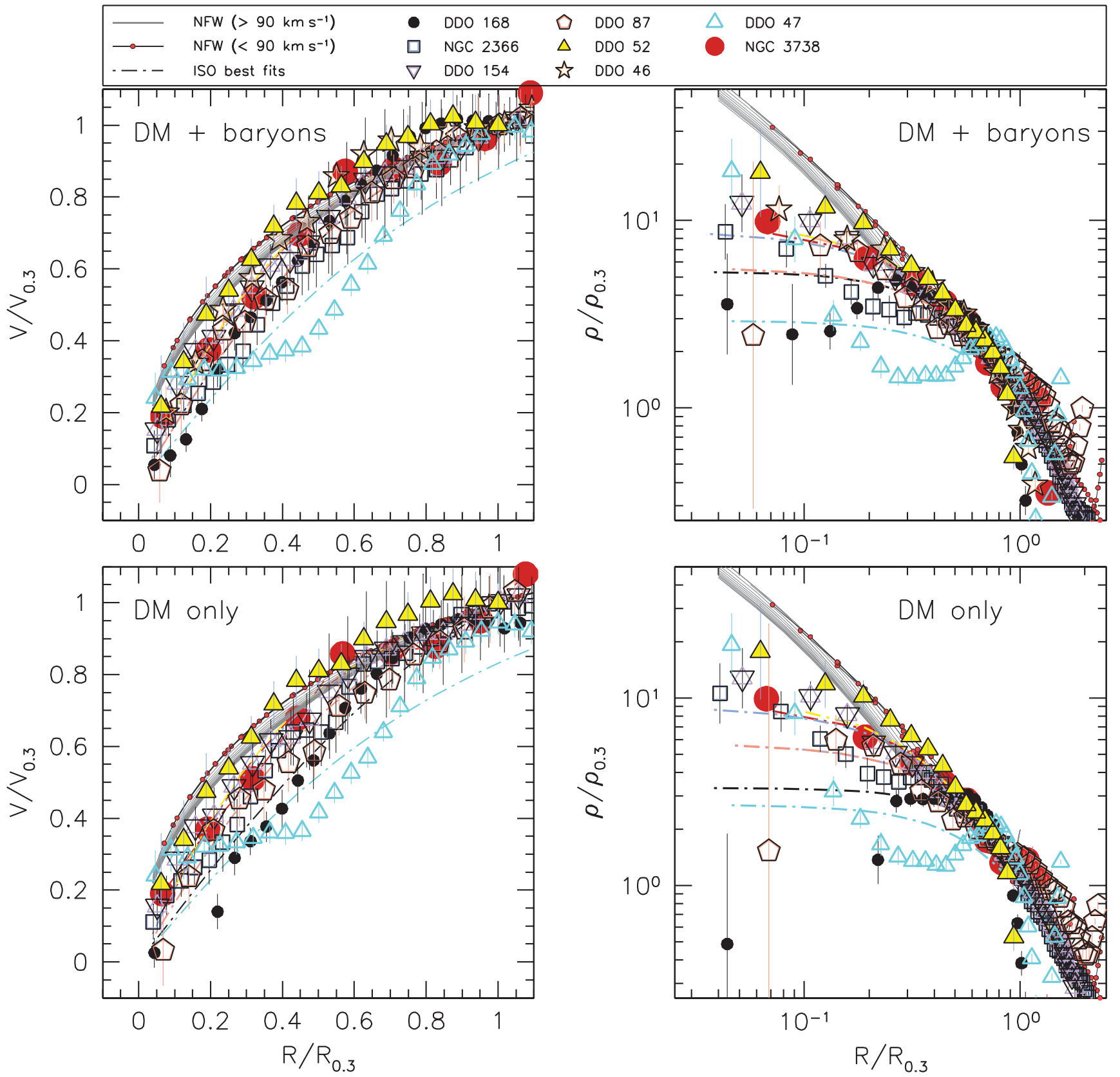

Figure 4. Left panels: the rotation curves of the remaining eight galaxies of the 26 LITTLE THINGS (in dynamical mass order) which are all scaled with respect to the rotation velocity $V_{03}$ at $R_{03}$ where the logarithmic slope of the rotation curve is $d \log V / d \log R=0.3$ as described in Hayashi \& Navarro (2006). The upper (DM + baryons) and lower (DM only) panels show the ones including and excluding the dynamical contribution by baryons, respectively. The gray solid and black solid lines with small dots indicate the CDM NFW dark matter rotation curves with $V_{200}$ which is $>90$ and $<90 \mathrm{~km} \mathrm{~s}^{-1}$, respectively. The dashed lines (denoted as $I S O$ ) show the best fitted pseudo-isothermal halo models to the galaxies. Right panels: the corresponding dark matter density profiles derived using the scaled rotation curves in the left panels. The gray $\left(V_{200}>90 \mathrm{~km} \mathrm{~s}^{-1}\right)$ and black solid lines with small dots $\left(V_{200}<90 \mathrm{~km} \mathrm{~s}^{-1}\right)$ represent the CDM NFW models with the inner density slope $\alpha \sim-1.0$. The dashed lines indicate the best fitted pseudo-isothermal halo models with $\alpha \sim 0.0$. See Section 4 for more details (continued).

The data used to create this figure are available.

the central cusps in their DM halos unlike the predictions from $\Lambda \mathrm{CDM}$ DM-only simulations.

\subsection{DM Density Profiles}

As a more direct way to examine the central DM distribution of the sample galaxies and compare them with $\Lambda \mathrm{CDM}$ simulations, we derive their DM density profiles from the DM rotation curves decomposed in Section 3.4. A direct conversion of the rotation curve to the corresponding DM density profile can be made by the following formula (see de
Blok et al. 2001 for more details),

$$
\rho(R)=\frac{1}{4 \pi G}\left[2 \frac{V}{R} \frac{\partial V}{\partial R}+\left(\frac{V}{R}\right)^{2}\right],
$$

where $V$ is a rotation velocity observed at a radius $R$, and $G$ is the gravitational constant. For this conversion, we assume a spherical halo potential which is valid for most nearby galaxies (Trachternach et al. 2008). This method has been used for deriving DM density profiles of dwarf and LSB disk galaxies, 


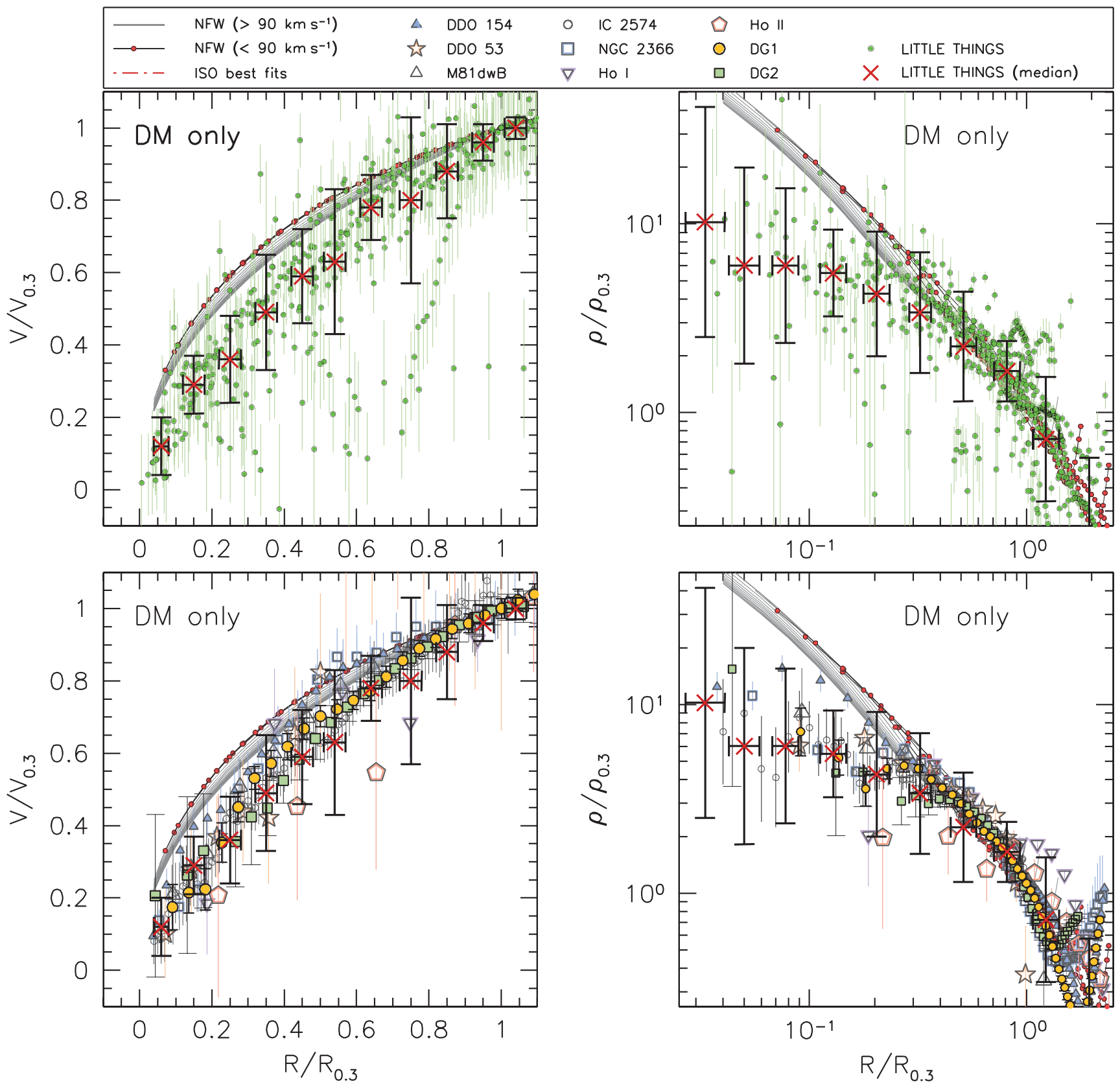

Figure 5. Upper-left panel: the (DM only) rotation curves (small dots) of the 21 LITTLE THINGS (including 3 THINGS galaxies) for which Spitzer3.6 $\mu$ m image is available. These are all scaled with respect to the rotation velocity $V_{03}$ at $R_{03}$ where the logarithmic slope of the rotation curve is $d \log V / d \log R=0.3$ as described in Hayashi \& Navarro (2006). The " $\times$ " symbol represents the median values of the rotation curves in each $0.1 \mathrm{R} / \mathrm{R}_{0.3}$ bin. The error bars show the $1 \sigma$ scatter. Lower-left panel: the scaled rotation curves of the seven THINGS, and the two simulated dwarf galaxies (DG1 and DG2 in Governato et al. 2010) which are overplotted to the median values of the LITTLE THINGS rotation curves. The gray solid and black solid lines with small dots indicate the CDM NFW dark matter rotation curves with $V_{200}$ which is $>90$ and $<90 \mathrm{~km} \mathrm{~s}^{-1}$, respectively. Right panels: the corresponding dark matter density profiles derived using the scaled rotation curves in the left panels. The gray $\left(V_{200}>90 \mathrm{~km} \mathrm{~s}^{-1}\right)$ and black solid lines with small dots $\left(V_{200}<90 \mathrm{~km} \mathrm{~s}^{-1}\right)$ represent the CDM NFW models with the inner density slope $\alpha \sim-1.0$. See Section 4 for more details.

and proved to be reliable as found in the comparison of the derived DM density profiles of simulated dwarf galaxies with their input ones (Oh et al. 2011a).

In the right panel of Figures 2-4, we present the derived (dark) matter density profiles of the LITTLE THINGS sample galaxies. In addition, we calculate the median values of the DM density profiles as shown in the upper-right panel of Figure 5. We also overplot the DM density profiles of the THINGS and simulated dwarf galaxies in the lower-right panel of Figure 5. The radial fluctuation shown in some of the DM density profiles (e.g., IC 1613 etc.) is largely due to fluctuations in the derived rotation curves at the relevant radius. These are mainly because of either the effect of non-circular motions, the noise in velocity profiles with low signal-to-noise ratio values, or both in the region.

As already implied by the solid-body like inner rotation curve shape of the LITTLE THINGS sample galaxies in Section 4.1, their central DM density profiles are systematically shallower than those of the cusp-like DM density profiles predicted from DM-only $\Lambda$ CDM simulations. As shown in the 
lower-right panel of Figures 2-4, the difference is even more pronounced in the comparison of the DM density profiles corrected for the baryons although the dynamical contribution by baryons is rather insignificant. The sample dIrr galaxies are more consistent with the core-like DM density profiles (dotdashed lines in the right-hand frames of Figures 2-4) than NFW-type profiles. This is much like the THINGS dwarf galaxies, and the simulated dwarfs (DG1 and DG2) with baryonic feedback processes as shown in the lower-right panel of Figure 5.

We also measure the inner density slopes $\alpha$ of the DM density profiles to quantify the cuspiness of the central DM distribution. This yields a more quantitative comparison between the observations and simulations. As shown in the figures in the appendix (e.g., panel (f) of Figure 10), we perform a least squares fit (dotted lines) to the inner data points (gray dots) within a "break radius." As described in de Blok \& Bosma (2002; see also Oh et al. 2011b), we determine a break radius of a DM density profile where the slope changes most rapidly in the inner region of the profile. Following de Blok \& Bosma (2002), we adopt the mean difference between the slopes which are measured including the first data point outside the break radius and excluding the data point at the break radius, respectively, as an error bar $\Delta \alpha$ of the inner density slope. We measure the inner density slopes $\alpha$, of the galaxies from their total matter (including both DM halo and baryons) as well as DM-only density profiles. The former, a so-called "minimum disk assumption" that attributes the total rotation curve to the DM component only, gives a steeper inner density slope. Meanwhile, the latter where the dynamical contribution by baryons is subtracted from the total rotation curve allows us to examine the effect of the model $Y_{\star}^{3.6}$ on the measured inner density slope. The mean values of the slopes of the 26 LITTLE THINGS dwarf galaxies are $\alpha_{\min }=-0.42 \pm 0.21$ and $\alpha_{Y_{\star}^{3.6}}=-0.32 \pm 0.24^{29}$ for the minimum disk and the model $r_{\star}^{3.6}$ disk assumptions, respectively.

As expected, the slopes measured assuming the minimum disk are slightly steeper than those measured using the model $r_{\star}^{3.6}$ disk for most sample galaxies. However, the difference between the two slopes is largely insignificant since most of the sample galaxies are DM dominated as indicated by their low baryonic fraction. The measured logarithmic inner density slopes $\alpha$ of our sample galaxies are listed in Table 2 .

The LITTLE THINGS sample dwarf galaxies do not in general agree with the steep logarithmic inner slope $(\sim 1.0)$ of the DM density profiles predicted from $\Lambda$ CDM DM-only simulations. Instead, they show a range of shallower slopes being consistent with a core-like DM distribution at the centers, which supports the previous results found in nearby dwarf and LSB disk galaxies. Our results $\left(\alpha_{\min }=-0.42 \pm 0.21\right.$; $\left.\alpha_{Y_{\star}^{3.6}}=-0.32 \pm 0.24\right)$ are consistent with the mean logarithmic slope, $\alpha_{\min }=-0.29 \pm 0.07$, of the seven THINGS dwarf galaxies derived assuming a minimum disk in $\mathrm{Oh}$ et al. (2011b). Moreover, if we combine the sample dwarf galaxies from LITTLE THINGS and THINGS which have a similar data quality and whose inner density slopes are derived in exactly the same way, the mean value of the slopes of the 29 dwarf galaxies is $\alpha_{\min }=-0.40 \pm 0.24$. This shows good agreement within the error bars with $\alpha_{\min }=-0.2 \pm 0.2$ derived from LSB

\footnotetext{
29 The dynamical contribution by the stellar component is included in the DM density profiles of five galaxies where no Spitzer $3.6 \mu \mathrm{m}$ image is available.
}

galaxies in de Blok et al. (2001) but a clear deviation from the $\alpha \sim-1.0$ predicted from $\Lambda$ CDM DM-only simulations.

A few galaxies in our sample, such as DDO 101 and DDO 210 whose rotation curves are equally fitted by both NFW and pseudo-isothermal halo models appear to have relatively steeper slopes compared to the other ones. However, as discussed in Section 3.4, this could be due to insufficient sampling of the DM density profiles in the inner region. The gradient of the logarithmic density slope, $d \log \rho / d \log R$ gradually decreases toward the outer region of a galaxy, giving a steeper slope $\alpha$. Therefore, the steeper a slope $\alpha$ is, the more data points in the outer regions are included when measuring the logarithmic slope of a DM density profile. This could, conceivably, be the case for galaxies with insufficient spatial sampling. As discussed above, the insufficient sampling mainly arises from either the smaller size of the H I disk (e.g., DDO 210) or the larger distances of the galaxies (e.g., Haro 36).

\section{EFFECT OF SN FEEDBACK ON THE CENTRAL CUSPS}

As discussed in Governato et al. (2010), the constant-density cores observed near the centers of dwarf galaxies can be reconciled with simulations by taking the effect of baryonic feedback processes into account without a need for any explicit modification of the current $\Lambda \mathrm{CDM}$ paradigm. In particular, repeated gas outflows driven by $\mathrm{SNe}$ have been found to be efficient enough to redistribute the matter in dwarf galaxies, resulting in shallower DM density profiles as observed in nearby dwarf galaxies (Oh et al. 2011a).

The investigation of the effect of SN-driven gas outflows on the central DM distribution has been extended to low mass field dwarf galaxies using GASOLINE (Wadsley et al. 2004), a parallel SPH tree-code with multistepping (Governato et al. 2012; Pontzen \& Governato 2012; di Cintio et al. 2014; see also Pontzen \& Governato 2014). In the simulations, the present-day stellar mass of galaxies ranges from $10^{9.8}$ down to $10^{4.5} M_{\odot}$ where the energy transfer from repeated gas outflows to the DM component becomes inefficient. More specifically, the SN feedback in small halos where less than $0.03 \%$ of the total amount of baryons is converted into stars is less effective at removing the central cusps and turning the cusp-like DM density profiles into core-like ones. According to this scenario, it is expected that the central DM distribution in these systems remains cuspy, and the inner slopes of their DM density profiles are steeper than those of higher mass counterparts (Governato et al. 2012; Zolotov et al. 2012). It implies that the central cusps predicted from $\Lambda \mathrm{CDM}$ DM-only simulations should survive in low mass halos. This underlines the cosmological importance of low mass dwarf galaxies in the local universe for testing the "cusp/core" problem.

As discussed earlier, the systematic uncertainties caused by low resolution radio observations are significantly reduced in the high-resolution $\mathrm{H}_{\mathrm{I}}$ data from LITTLE THINGS, which allows us to derive more accurate rotation curves of the sample galaxies and thus their central DM distributions. In addition, the Spitzer IRAC $3.6 \mu \mathrm{m}$ data combined with model $\Upsilon_{\star}^{3.6}$ values based on stellar population synthesis models and galaxy colors provide more reliable stellar masses of the sample galaxies.

In Figure 6, we plot the inner density slopes $\alpha$ of the sample dwarf galaxies from both LITTLE THINGS and THINGS against their stellar masses $M_{*}$ on a logarithmic scale. In addition, we also add those of a sample of THINGS disk galaxies whose mass models were derived in de Blok et al. 


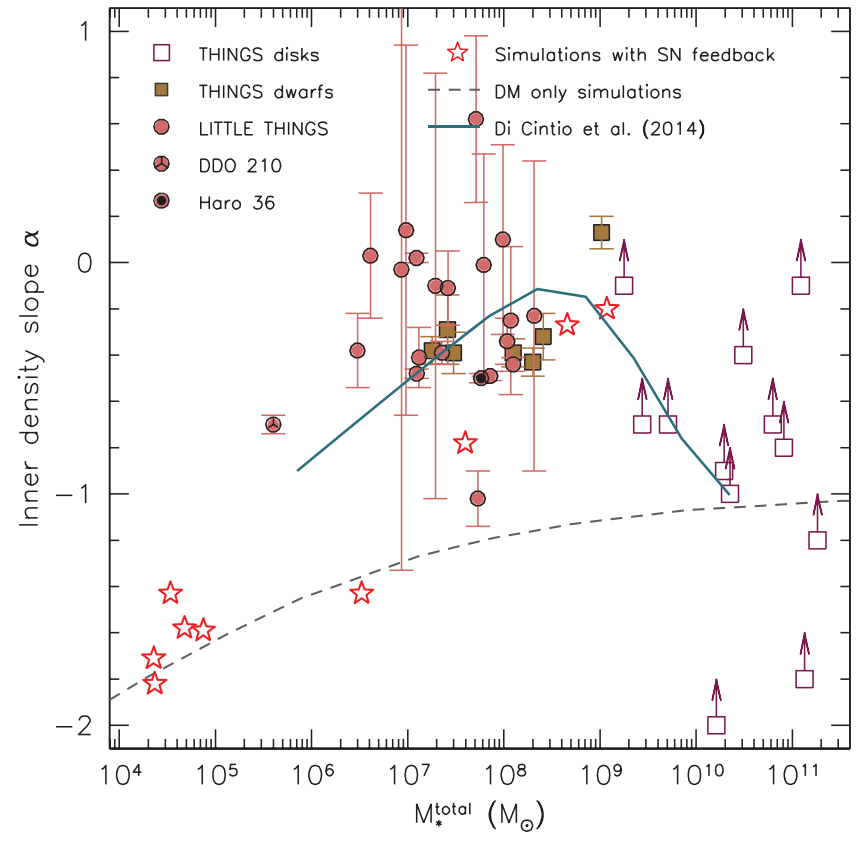

Figure 6. Inner dark matter density slope $\alpha$ of the sample galaxies from LITTLE THINGS (filled circles) and THINGS (filled squares, THINGS dwarfs; open squares, THINGS disk galaxies) against their total stellar masses. The dashed line indicates the $\alpha-M_{*}^{\text {total }}$ prediction from $\Lambda$ CDM dark matter only simulations. The open stars and solid line represent the $\alpha \mathrm{vs} . \mathbf{M}_{*}^{\text {total }}$ of the resolved halos from the $\Lambda \mathrm{CDM}$ SPH simulations with baryonic feedback processes which are measured at $500 \mathrm{pc}$ and $z=0$ (Governato et al. 2012; di Cintio et al. 2014). See Section 5 for more discussions.

(2008) in order to examine the $\alpha-M_{*}$ relationship in the higher mass regime. Compared to dwarf galaxies, the central kinematics of disk galaxies are usually dominated by a bulge component. It is therefore not trivial to perform a reliable diskhalo decomposition of the disk galaxies despite using the multiwavelength data from THINGS whose data quality is comparable to that of LITTLE THINGS. For this reason, in Figure 6, we use the inner density slopes of the disk galaxies measured assuming a minimum disk. As discussed in Section 4.2, it should be noted that the minimum disk assumption attributes the total kinematics of a galaxy to the DM only, ignoring the contribution of baryonic components, giving a lower limit on the inner density slope (i.e., a steeper slope).

As shown in Figure 6, the $\Lambda$ CDM SPH simulations including the effect of baryonic feedback processes predict cusp-like DM distributions with steeper inner density slopes $(\alpha<-1.0)$ in DM halos whose stellar mass is less than about $10^{6} M_{\odot}$. As discussed in Governato et al. (2012), the lower the mass of the stellar component in a galaxy, the less the dynamical effect of $\mathrm{SN}$ feedback on the DM potential. Consequently, this results in the central DM distribution in the low mass halo regime remaining cuspy. According to this, the initial cusps formed in the early universe would still exist today in low mass dwarf galaxies which have stellar masses less than $\sim 10^{6} M_{\odot}$ where the repeated thermal energy injection from SN explosions becomes substantially inefficient, mainly due to the rapidly decreasing star formation efficiency in these systems (Governato et al. 2012; Pontzen \& Governato 2012).

However, in Figure 6, as the stellar mass of a galaxy increases, the inner DM density slope $\alpha$ becomes shallower in the $\Lambda$ CDMSPH simulations including baryonic feedback processes (open stars) with respect to the counterpart in the DM-only simulations (dashed line). As discussed in Governato et al. (2010) and Pontzen \& Governato (2012) (see also di Cintio et al. 2014), the central DM cusps can be disrupted by the rapid gas injection caused by $\mathrm{SN}$-driven gas outflows into the central region of galaxies, resulting in a shallower DM density distribution. This shows that DM-baryon interactions in dwarf galaxies through gas outflows play a critical role not only in forming bulgeless dwarf galaxies but also in flattening central cusps predicted from DM-only $\Lambda$ CDM simulations. This demonstrates that proper modeling of DM-baryon interactions in hydrodynamical $\Lambda \mathrm{CDM}$ galaxy simulations is able to alleviate the long-standing tension associated with the central DM distribution in dwarf galaxies between simulations and observations. We refer to Governato et al. (2012) (see also Pontzen \& Governato 2012, 2014; and di Cintio et al. 2014) for a detailed discussion of the effect of SN feedback on the central DM distribution in dwarf galaxies.

Meanwhile, in Figure 6, the trend of slope change $d \log \alpha / d \log M_{*}$ predicted from dwarf galaxy simulations with baryonic feedback processes is reversed in massive disk galaxies (open boxes) where a bulge component becomes dominant in the central kinematics. As discussed in di Cintio et al. (2014), the effect of SN feedback can be surpassed by the deep gravitational potential which is caused by the bulge component in the central region. However, as discussed earlier, the steep slopes of the THINGS disk galaxies could be partially affected by the minimum disk assumption used for deriving their DM density profiles. The central kinematics of a disk galaxy with a substantial bulge component is sensitive to even small uncertainties in $Y_{\star}$ when converting the luminosity profile to the mass density profile. This makes it difficult to perform an accurate disk-halo decomposition of bulge-dominated disk galaxies. This stresses the usefulness of bulge-less dwarf galaxies in testing the effect of SN feedback on the central DM distribution of galaxies.

As shown in Figure 6, the simulated dwarf galaxies which have comparable stellar masses ranging from $10^{6}$ to $10^{9} M_{\odot}$ show good agreement with the majority of the sample dwarf galaxies from THINGS and LITTLE THINGS. However, as already discussed in Section 4.2, the rotation curves of some LITTLE THINGS sample galaxies (e.g., DDO 70, DDO 101, DDO 154, DDO 210 and Haro 36) are equally well fitted by CDM NFW and pseudo-isothermal halo models in terms of $\chi_{\text {red }}^{2}$ values. Moreover some of them, such as DDO 101, DDO 210 and Haro 36, appear to have relatively steep inner density slopes (although Haro 36 is defined as a blue compact dwarf galaxy which usually shows a steep increase in rotation velocity in the inner region mainly due to young starburst components formed during galaxy interaction or mergers; e.g., Bekki 2008). In particular, DDO 210 is a good candidate for testing the SN feedback efficiency scenario by Governato et al. (2012) given that its stellar mass falls within the regime where according to the simulations primordial CDM cusps are expected to survive. In Figure 6, DDO 210 shows no distinct signature of the central cusp $(\alpha \sim-1.0)$ given its corresponding stellar mass, deviating from the prediction of $\Lambda$ CDM DMonly simulations (dashed line). Nevertheless, in a qualitative sense, its relatively steep inner density slope $\alpha \sim-0.70$ compared to the others still leaves a room for a potential signature of the central cusp, which supports the lower SN energy injection scenario in low mass dwarf galaxies. 


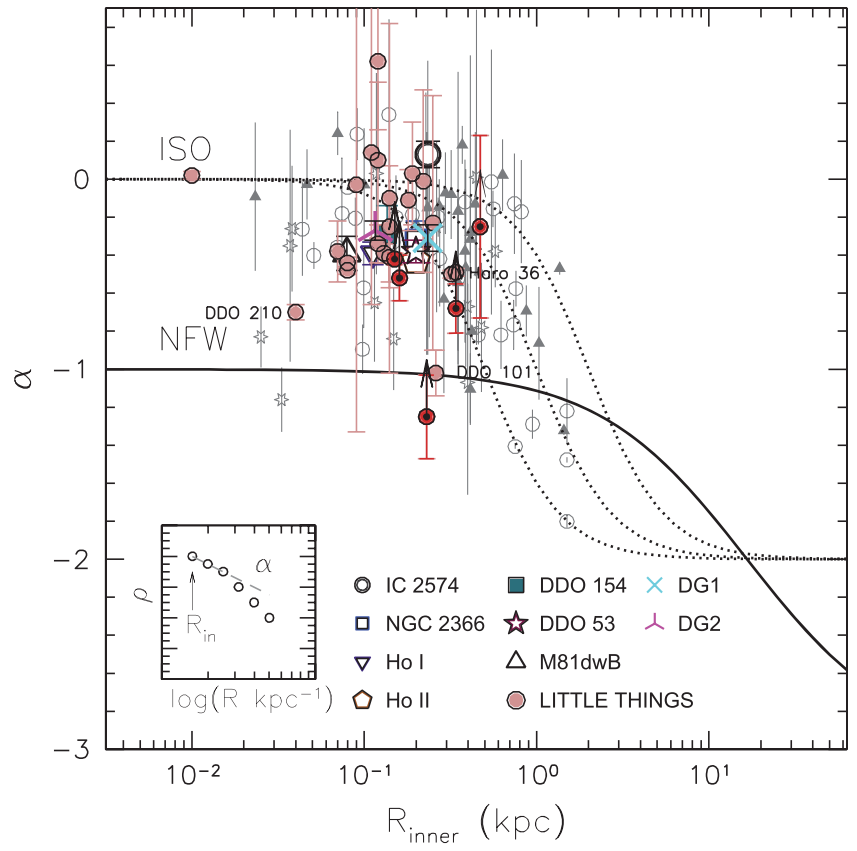

Figure 7. Inner slope of the dark matter density profiles $\alpha$ vs. the radius $R_{\text {in }}$ of the innermost point within which $\alpha$ is measured as described in the small figure (de Blok et al. 2001). The $\alpha-R_{\text {in }}$ of the sample galaxies from LITTLE THINGS, THINGS and the two simulated dwarf galaxies (DG1 and DG2: Governato et al. 2010) as well as the previous measurements (gray symbols) of LSB galaxies (open circles: de Blok et al. 2001; triangles: de Blok \& Bosma 2002; open stars: Swaters et al. 2003). Filled circles with arrows indicate the galaxies of which inner density slopes are measured assuming a "minimum disk," giving a steeper slope. The solid and dotted lines represent the $\alpha-R_{\text {in }}$ trends of dark-matter-only $\Lambda$ CDM NFW and pseudo-isothermal halo models, respectively. See Section 6 for more details.

However, as noted earlier, the resolution of LITTLE THINGS $\mathrm{H}$ i observations is not high enough to resolve the small $\mathrm{H}$ i disk $\left(\sim 60^{\prime \prime}\right.$ diameter $)$ and distinguish between cusp- and core-like DM behavior near the center of DDO 210. We note that DDO 210 has the most compact $\mathrm{H}_{\text {I }}$ disk (in terms of the beam size, e.g., $R_{\text {max }} \mathrm{HI}_{\text {beam }}^{-1} \sim 6.0$ as given in Table 2) of the sample galaxies. Likewise, some of our sample galaxies may still suffer from beam smearing which is discussed in the following section.

\section{INNER DENSITY SLOPE VERSUS RESOLUTION}

As in de Blok et al. (2001), for a quantitative examination of the beam smearing effect on our sample galaxies, we plot the inner density slopes $\alpha$ of the galaxies including the THINGS sample as well as the two simulated dwarf galaxies (DG1 and DG2) modeled by Governato et al. (2010) against the observed radii of their innermost point $R_{\text {inner }}$ in Figure 7 . For the sample dwarf galaxies from LITTLE THINGS, we use the slopes derived assuming the model $Y_{\star}^{3.6}$ disk. We also show the $\alpha$ $R_{\text {inner }}$ relations of the NFW and pseudo-isothermal halo models as solid and dotted lines, respectively, derived using their analytical formulas as given in Equations (6) and (8).

As shown in Figure 7, most sample galaxies show significant deviations from the predicted $\alpha-R_{\text {inner }}$ trend (solid line) of $\Lambda \mathrm{CDM}$ NFW halos at around a $R_{\text {inner }}$ of $\sim 0.2 \mathrm{kpc}$. Instead, they are more consistent with those of pseudo-isothermal halo models with different core-radii (dotted lines) as well as the earlier results found from LSB galaxies (gray symbols) in de Blok \& Bosma (2002). However, the clear difference between the two halo models (i.e., NFW and pseudo-isothermal) at high resolutions (e.g., $R_{\text {inner }}<0.5 \mathrm{kpc}$ ) becomes ambiguous as the innermost radius $R_{\text {inner }}$ of a given DM density profile increases. For example, a galaxy with a larger $R_{\text {inner }}$ (i.e., low resolution) tends to show a steeper inner slope of DM density profile. The larger $R_{\text {inner }}$ makes it lie in the region where the slopes of the two halo models are approximately similar to each other. In addition, as discussed in Section 4.2, the derivative $d \log \rho / d \log R$ of a DM density profile on a logarithmic scale decreases toward the outer region of a galaxy. If the DM density profile is affected by beam smearing, the break radius of the profile which is determined when measuring the inner slope tends to migrate into the outer regime where $d \log \rho / d \log R$ has a lower value. Therefore, the inner density slope $\alpha$ within the break radius is most likely to be steeper than the ones derived from well sampled profiles. This could be the case of DDO 101 and DDO 210. In particular, DDO 101 is most likely to be affected by the beam smearing effect as shown in Figure 7.

Yet higher resolution velocity fields obtained with radio interferometers or using other tracers such as integral field mapping are required to study the effect of SN feedback on the central cusps of the lowest mass dwarf galaxies. Such highresolution observations of low mass dwarf galaxies would provide an ultimate test of the $\Lambda \mathrm{CDM}$ paradigm. Unlike clusters of galaxies where the depth of the gravitational potential well is deep enough to retain warm DM (WDM) as well as CDM, there is no room for WDM in dwarf galaxies inhabiting DM halos with much shallower potential wells. Therefore, finding a signature of a central cusp in dwarf galaxies will prove that there is at least some CDM in the universe. This again highlights the cosmological importance of low mass dwarf galaxies, not only for resolving the "cusp/core" controversy in $\Lambda \mathrm{CDM}$ simulations but also as an indirect proof for the existence of CDM in the universe.

\section{CONCLUSION}

In this paper we derive the rotation curves of 26 dwarf galaxies culled from LITTLE THINGS, and examine their DM distributions near the centers of the galaxies. From this, we address the "cusp/core" problem which has been one of the long-standing problems in $\Lambda \mathrm{CDM}$ simulations on galactic scales. The high-resolution LITTLE THINGS H I data $\left(\sim 6^{\prime \prime}\right.$ angular; $\sim 2.6 \mathrm{~km} \mathrm{~s}^{-1}$ spectral) complemented with optical and Spitzer IRAC $3.6 \mu \mathrm{m}$ images are sufficiently detailed to resolve the central region of the sample galaxies where the cusp- and core-like halo models are clearly distinguished.

In particular, we use the bulk velocity fields of the galaxies extracted using the method described in Oh et al. (2008) to correct for turbulent random non-circular gas motions. This enables us to derive more reliable rotation curves and thus more accurate DM distributions in the galaxies. We corrected for the modest dynamical contribution by baryons in dwarf galaxies by using Spitzer IRAC $3.6 \mu \mathrm{m}$ images combined with model $\Upsilon_{\star}^{3.6}$ values based on stellar population synthesis models. This allowed us to derive robust mass models of the stellar components of the galaxies and thus better constrain their central DM distributions.

From this, we found that the decomposed DM rotation curves of most sample galaxies are well matched in shape to those of core-like halos which are characterized by a linear increase of rotation velocity in the inner region. We also derive 
the DM density profiles of the sample galaxies and quantify the degree of the central DM concentration by measuring the logarithmic inner slopes of the profiles. The mean value of the inner slopes $\alpha$ of the 26 sample galaxies is -0.32 , which indicates a mass distribution with a sizable constant densitycore toward the centers of the galaxies. This is consistent with that found in most nearby dwarf galaxies (e.g., LSB galaxies in de Blok \& Bosma 2002; THINGS dwarfs in Oh et al. 2011b) which all show a linear increase in the inner shapes of their rotation curves resulting in shallower inner density slopes $(\langle\alpha\rangle \sim-0.2)$. Considering the fact that observational uncertainties are significantly reduced in the high-resolution LITTLE THINGS data, the core-like DM distribution found in our sample galaxies provides a stringent observational constraint on the central DM distribution of halos in $\Lambda$ CDM simulations.

We find that the derived slopes of the DM density profiles do not agree with the cusp predicted by $\Lambda$ CDMDM-only simulations. However, recent cosmological $N$-body SPH galaxy simulations by Governato et al. (2010) (see also Governato et al. 2012; Pontzen \& Governato 2012) have shown that the discrepancy between observations and simulations can be reconciled within the $\Lambda \mathrm{CDM}$ paradigm by considering the dynamical effect of baryonic feedback processes on the central cusps. According to the simulations, DM-baryon interactions in dwarf galaxies through gas outflows driven by $\mathrm{SN}$ explosions play a critical role not only in forming bulgeless dwarf galaxies but also in turning central cusps into cores.

As discussed in Oh et al. (2011a), the slowly rising rotation curves and the resulting shallower DM density profiles of the simulated dwarf galaxies with $\mathrm{SN}$ feedback are qualitatively similar to those of the dwarf galaxies from THINGS. This is also the case for our sample galaxies from LITTLE THINGS whose DM rotation curves in the inner region rise too slowly to match the steep rotation curves of CDM halos. This shows that proper modeling of DM-baryon interactions in $\Lambda \mathrm{CDM}$ galaxy simulations is able to alleviate the long-standing tension between observations and simulations regarding the central DM distribution in dwarf galaxies.

Notwithstanding the dominant trend of core-like DM distribution in the LITTLE THINGS sample galaxies, some of the sample galaxies, such as DDO 210 and Haro 36 are equally well fitted by core- and cusp-like halo models in describing their DM rotation curves. They have relatively steeper inner density slopes with $\alpha \approx-0.70$ and -0.50 for DDO 210 and Haro 36, respectively, compared to the mean value (DM only) of the rest of the sample $(\sim-0.29)$. It is possible, however, despite the high angular resolution of the LITTLE THINGS $\mathrm{H}_{\mathrm{I}}$ data, the inner density slopes of these two galaxies are affected by beam smearing (the $\mathrm{H}$ i disk of DDO 210 being intrinsically small and Haro 36 being one of the more distant dwarfs). Insufficient spatial resolution in the inner region of the galaxies results in steeper observed inner DM density slopes.

According to the latest $N$-body SPH simulations of dwarf galaxies with baryonic feedback processes (Governato et al. 2012), the SN feedback in low mass dwarf galaxies with a stellar mass less than $10^{6} M_{\odot}$ is not sufficient to disrupt the central cusps, the repeated energy injection from SN explosions into DM halos becoming inefficient, largely due to low star formation efficiencies in these low mass systems. However, previous observational studies regarding the "cusp/core" problem have mostly focused on relatively massive dwarf galaxies for which reliable rotation curves are available. Low mass dwarf galaxies have been usually excluded for the study of the central DM distribution due to the low amplitude of their maximum rotation velocities. The kinematics of such low mass dwarf galaxies is more vulnerable to kinematic disturbances like non-circular motions in galaxies compared to more massive ones, and also more sensitive to additional corrections (e.g., asymmetric drift) made when deriving rotation curves. In this respect, the possibility of a selection effect in favor of relatively massive dwarf galaxies where the effect of $\mathrm{SN}$ feedback is enough to turn the central cusps into cores should be considered.

It would therefore be worthwhile to perform high-resolution follow-up observations, for example using an optical integral field unit, of low mass dwarf galaxies including some of the LITTLE THINGS dwarf galaxies (e.g., DDO 210) whose stellar masses lie in the regime where primordial CDM cusps are predicted from the simulations (Governato et al. 2012). These high-resolution observations will enable us to achieve a finer sampling of the central region of the galaxies, and thus more accurate inner DM density profiles. From this, more stringent observational constraints on the central cusp of low mass dwarf galaxies could be provided. Moreover, an accurate measurement of the DM distribution in these low mass dwarf galaxies will provide an ultimate test for the CDM paradigm given that dwarf galaxies inhabiting DM halos with a shallow potential well have only room for CDM, unlike clusters of galaxies whose gravitational potential is deep enough to retain WDM as well as CDM. Therefore, the presence or absence of a signature of the central cusp in these low mass halos will provide a critical observational test, either supporting or falsifying the $\Lambda \mathrm{CDM}$ paradigm.

We thank W. J. G. de Blok for providing the inner density slopes and stellar masses of the THINGS disk galaxies and useful comments and discussion. We thank Fabio Governato, Andrew Pontzen, Chris Brook and Arianna Di Cintio for useful discussion on the simulations and providing the data. Parts of this research were conducted by the Australian Research Council Centre of Excellence for All-sky Astrophysics (CAASTRO), through project number CE110001020. This work was funded in part by the National Science Foundation through grants AST-0707563 and AST-0707426 to D. A. H. and B. G. E.

\section{APPENDIX A}

\section{DATA AND KINEMATIC ANALYSIS}

In this appendix, we present the data and kinematic analysis of the 26 LITTLE THINGS (Hunter et al. 2012) dwarf galaxies. For each galaxy, we show the (1) data (Figures 11, $14,17,20,23,26,29,32,35,38,41,44,47,50,53,56,59,62$, $65,68,71,74,77,80,83$, and 86), (2) kinematic analysis (Figures 12, 15, 18, 21, 24, 27, 30, 33, 36, 39, 42, 45, 48, 51, $54,57,60,63,66,69,72,75,78,81$, and 84), and (3) mass modeling $(13,16,19,22,25,28,31,34,37,40,43,46,49,52$, $55,58,61,64,67,70,73,76,79$, and 85) with descriptions.

A. Data-(a) integrated $\mathrm{H}_{\mathrm{I}}$ intensity map (moment 0 ). The contour levels start at $+3 \sigma$ in steps of $+3 \sigma$. (b) IWM velocity field (moment 1). (c) Velocity dispersion map (moment 2). (d) Spitzer IRAC $3.6 \mu \mathrm{m}$ image obtained from the archives including "SINGS" (Kennicutt et al. 2003) and Spitzer "Local Volume Legacy" (Dale et al. 2009). (e) Bulk velocity field 